\title{
Theoretical Determination of Binding Energies of Small Molecules on Interstellar Ice Surfaces
}

\author{
Denis Duflot *, Céline Toubin * and Maurice Monnerville \\ Univ. Lille, CNRS, UMR 8523 - PhLAM - Physique des Lasers Atomes et Molécules, Lille, France
}

\section{OPEN ACCESS}

Edited by:

Piero Ugliengo,

University of Turin, Italy

Reviewed by:

Feliu Maseras,

Institut Català d'Investigació Química,

Spain

Albert Rimola,

Autonomous University of Barcelona,

Spain

*Correspondence:

Céline Toubin

celine.toubin@univ-lille.fr

Denis Duflot

denis.duflot@univ-lille.fr

Specialty section:

This article was submitted to

Astrochemistry,

a section of the journal

Frontiers in Astronomy and Space

Sciences

Received: 22 December 2020

Accepted: 08 February 2021

Published: 26 March 2021

Citation:

Duflot D, Toubin C and Monnerville $M$ (2021) Theoretical Determination of Binding Energies of Small Molecules on Interstellar Ice Surfaces.

Front. Astron. Space Sci. 8:645243. doi: 10.3389/fspas.2021.645243
The adsorption of a series of atoms and small molecules and radicals $(\mathrm{H}, \mathrm{C}, \mathrm{N}, \mathrm{O}, \mathrm{NH}, \mathrm{OH}$, $\mathrm{H}_{2} \mathrm{O}, \mathrm{CH}_{3}$, and $\mathrm{NH}_{3}$ ) on hexagonal crystalline and amorphous ice clusters were obtained via classical molecular dynamics and electronic structure methods. The geometry and binding energies were calculated using a QMHigh:QMLow hybrid method on model clusters. Several combination of basis sets, density functionals and semi-empirical methods were compared and tested against previous works. More accurate binding energies were also refined via single point Coupled Cluster calculations. Most species, except carbon atom, physisorb on the surface, leading to rather small binding energies. The carbon atom forms a $\mathrm{COH}_{2}$ molecule and in some cases leads to the formation of a $\mathrm{COH}-\mathrm{H}_{3} \mathrm{O}^{+}$complex. Amorphous ices are characterized by slightly stronger binding energies than the crystalline phase. A major result of this work is to also access the dispersion of the binding energies since a variety of adsorption sites is explored. The interaction energies thus obtained may serve to feed or refine astrochemical models. The present methodology could be easily extended to other types of surfaces and larger adsorbates.

Keywords: interstellar, ices, binding energies, theoretical, amorphous, interstellar medium

\section{INTRODUCTION}

Astrochemistry has been an area of intense research in particular with the aim to elucidate the origin of the chemical diversity observed in space. Depending on the evolution stage of the interstellar dust and the region, energetic processes may dominate the chemistry. However, in low UV-photon field regions, as in dense molecular clouds, energetic processes are suppressed and surface reactions of cold molecules become important. It has been demonstrated both experimentally and theoretically that dust grains catalyze the formation of some important molecules, such as $\mathrm{H}_{2}, \mathrm{H}_{2} \mathrm{O}$, and $\mathrm{CO}_{2}$, organic molecules $\left(\mathrm{H}_{2} \mathrm{CO}, \mathrm{CH}_{3} \mathrm{OH} \ldots\right.$ ) (Ioppolo et al., 2011; Pirim and Krim, 2011; Peters et al., 2013; Rimola et al., 2014; Minissale et al., 2015), or complex/prebiotic molecules (Koch et al., 2008; Rimola and Ugliengo, 2009). Thermally activated surface reactions rarely occur over the activation energy barriers on ice mantles because of their low temperatures $(\sim 10 \mathrm{~K})$. In contrast, quantumtunneling reactions are facilitated in this temperature range. The importance of tunneling effects has been discussed in various studies (Goumans and Kästner, 2010; Hama and Watanabe, 2013; Hickson et al., 2016). Whatever the conditions are, for a reaction to occur on the grain, the molecular species needs to reside or diffuse at the grain surface. Most neutral species can physisorb onto grains through van der Waals interactions at the low temperature conditions of dense molecular clouds. Chemisorption may also occur for radicals or reactive molecules on specific or defective sites. In contrast to chemisorption, physisorption is reversible, the desorption rate being derived from the 
binding energy and the frequency characterizing the motion of the adsorbed species in the potential energy well. Diffusion is also to be considered when reactions or recombinations occur through Langmuir-Hinshelwood mechanisms. Most astrochemical models (Garrod et al., 2006; Taquet et al., 2013; Ruaud et al., 2016; Cuppen et al., 2017; Wakelam et al., 2017) mimicking the chemical evolution in star forming region have now integrated these gas-grain processes. Among the molecular data used as inputs to these models, the adsorption energy is a key parameter, the diffusion energy being estimated as a fraction (between 0.3 and 1.0) of the binding energy (Ruaud et al., 2016). There is thus a crucial need to improve the binding energies (BE's) values in the models to better reproduce the astrophysical abundances.

Binding energies depend indeed on the nature of the volatile species, the surface and its coverage. BE's can be determined experimentally for stable molecules using programmed desorption (TPD) methods (Burke and Brown, 2010; Hama and Watanabe, 2013). Several studies have reviewed known binding energies on various surfaces and more especially water ices (Fraser et al., 2001; Collings et al., 2004; Noble et al., 2012; Hama and Watanabe, 2013; Penteado et al., 2017; Wakelam et al., 2017). However, TPD is not appropriate for reactive atoms or radicals, since they may spontaneously recombine to form stable molecules at the surface before thermal desorption. Consequently, binding energies of atoms or radicals are less documented.

For species with undefined BE's, attempts have been made by speculating an additive law: the $\mathrm{BE}$ of a given molecule being the sum of the BE's of the atoms or groups of atoms. This approximation of the $\mathrm{BE}$ as implemented in Garrod et al.'s model (Cuppen et al., 2017) gives a rough estimation that can indeed be improved in different manners. Wakelam et al. (Wakelam et al., 2017) have proposed a proportional law that relates the interaction energy between the given species and one water molecule (energy of the dimer) and its binding energy on amorphous solid water. However, the integration of the binding energies computed with this method in the gas-grain code Nautilus (Ruaud et al., 2016) did not improve the prediction of the gas phase abundances. A more precise determination of the BEs and inclusion of nonthermal processes have been invoked as possible improvements. Because of their accuracy, electronic structure calculations appear to be the method of choice to improve the $\mathrm{BE}$ determination. However, for computational reasons, this approach often reduces the explicit description of the surface to a small number of atoms or molecules (Koch et al., 2007; Duvernay et al., 2014; Rimola et al., 2014). Thanks to the development of numerical resources, periodic $a b$ initio simulations have been also performed recently to account for larger crystalline ice surfaces though still in a static way neglecting temperature effects and the configurations sampling being limited to the identification of the global minimum (Ferrero et al., 2020a). It should be noted that the size of the crystal and the choice of the functional are critical to surely assess an accurate value of the binding energy (Ferrero et al., 2020b). By contrast, semi-empirical based classical molecular dynamics (MD) simulations account at low computational cost for long range effects through the modeling of quasi-infinite surfaces and offer a full exploration of the possible binding sites providing $\mathrm{BE}$ distributions and eventually diffusion barriers (Michoulier et al., 2018). However, classical MD rely strongly on the accuracy, or even availability, of force-field parameters. Such parameters are in particular not available for radicals. Hybrid QM/MM (Quantum Mechanics/Molecular Mechanics) approaches, such as the ONIOM method (Chung et al., 2015), recently used by Sameera et al. (Sameera et al., 2017; Sameera and Maseras, 2018) on crystalline water ices, have the advantage of describing accurately the adsorbate-surface interaction including long range effects, dividing the system into two subunits: the adsorbate and few molecules of the surface treated at a high QM level and the rest of the surface being treated with a MM or lower level QM' approach. The latter $\mathrm{QM} / \mathrm{QM}$ ' scheme is the methodology chosen in the present study on ice surfaces generated from classical molecular dynamics simulations, including amorphous ice samples, to quantify the effect of structural disorder.

The binding energy depends on the volatile but also on the grain surface composition. Numerous studies have been conducted to determine the composition of the grains and more especially the grain icy mantle (Whittet et al., 1996; van Dishoeck, 1998; Ehrenfreund and Charnley, 2000; Ehrenfreund et al., 2003; Pontoppidan et al., 2008). They revealed that interstellar ices are mainly composed of water, but may also contain other species like $\mathrm{CO}, \mathrm{CO}_{2}, \mathrm{NH}_{3}$, and methanol, in less significant quantities. The precise structure of interstellar ice remains an open question, though it is generally admitted that it might be amorphous (Dulieu et al., 2013; Hama and Watanabe, 2013). Amorphous Solid Water (ASW) is known to have physical properties that are distinct from other crystalline states, e.g., a lower thermal conductivity, as well as a larger surface area and higher porosity (Dohnálek et al., 2003). In the spectrum of ASW, a small feature is present on the high wavenumber wing of the $\mathrm{OH}$ stretching modes band, due to $\mathrm{OH}$ dangling bonds, that are more abundant in the microporous material than in crystalline compact ice (Noble et al., 2014). These unsaturated sites are preferential adsorption sites for incoming molecules (Buch and Devlin, 1991; Michoulier et al., 2020). Proton disorder in amorphous ice was shown to trigger a diversity of adsorption sites and consequently of binding energies for small adsorbates compared to crystalline ice (Kimmel et al., 2001; Kimmel et al., 2001). Even though laboratory ASW or ice grown in MD simulations do not reproduce interstellar icy grains for various factors (growth conditions, porosity, size and time scales ... ) (Hama and Watanabe, 2013), characterizing the BEs of various molecules on these model surfaces provide nonetheless valuable insights on the trapping of the gas-phase species, on their availability for surface reactions and on the influence of the surface heterogeneity on the $\mathrm{BE}$ values.

The purpose of the present study is to establish a theoretical framework combining a force field based MD sampling and density functional corrected with high level $a b$ initio calculations on some configurations issued from this sampling to accurately describe binding energies (physisorption or chemisorption) of a set of atoms, radicals or molecules on 


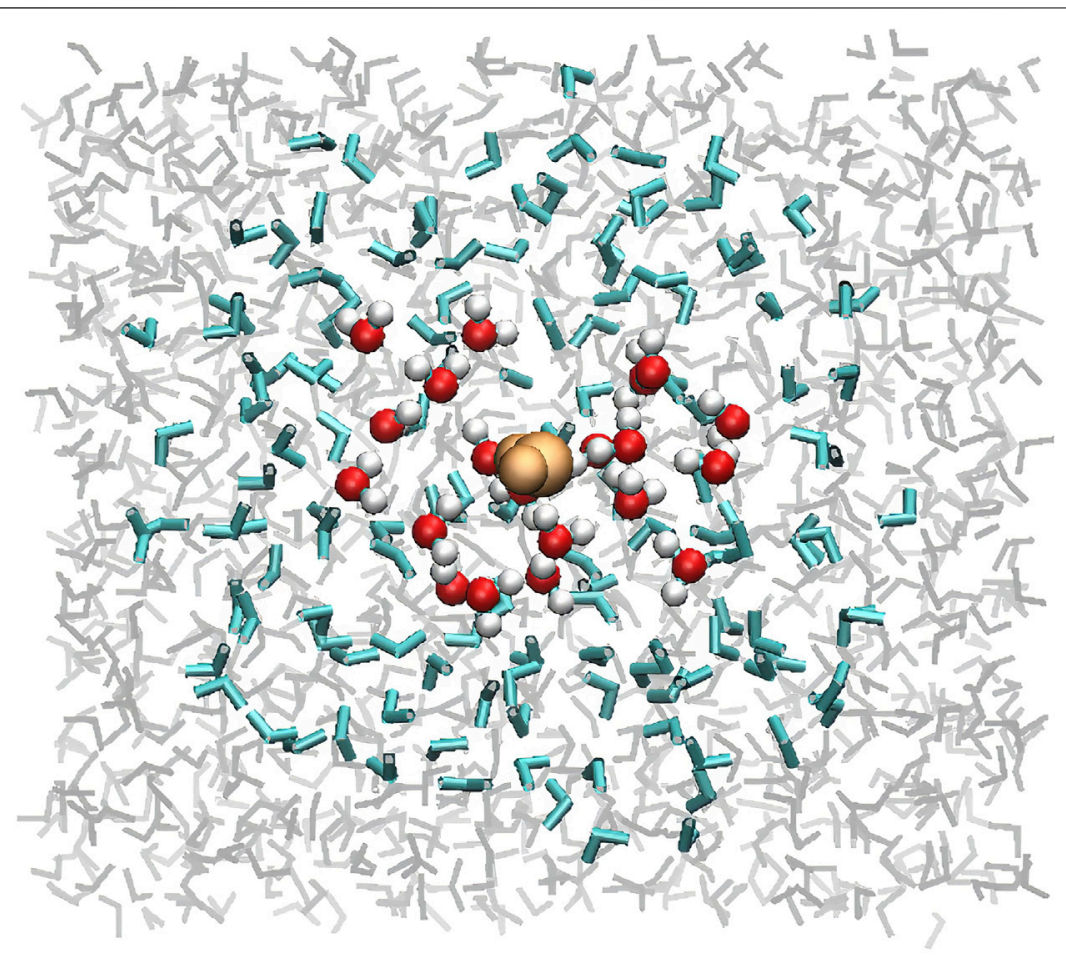

FIGURE 1 | Top view of a snapshot of amorphous ice issued from a MD trajectory at $77 \mathrm{~K}$. The layers for the ONIOM calculation are represented accordingly: adsorbed $\mathrm{H}_{2} \mathrm{O}$ (beige), QMHigh (red/white), QMLow (blue), and the remaining water molecules (grey).

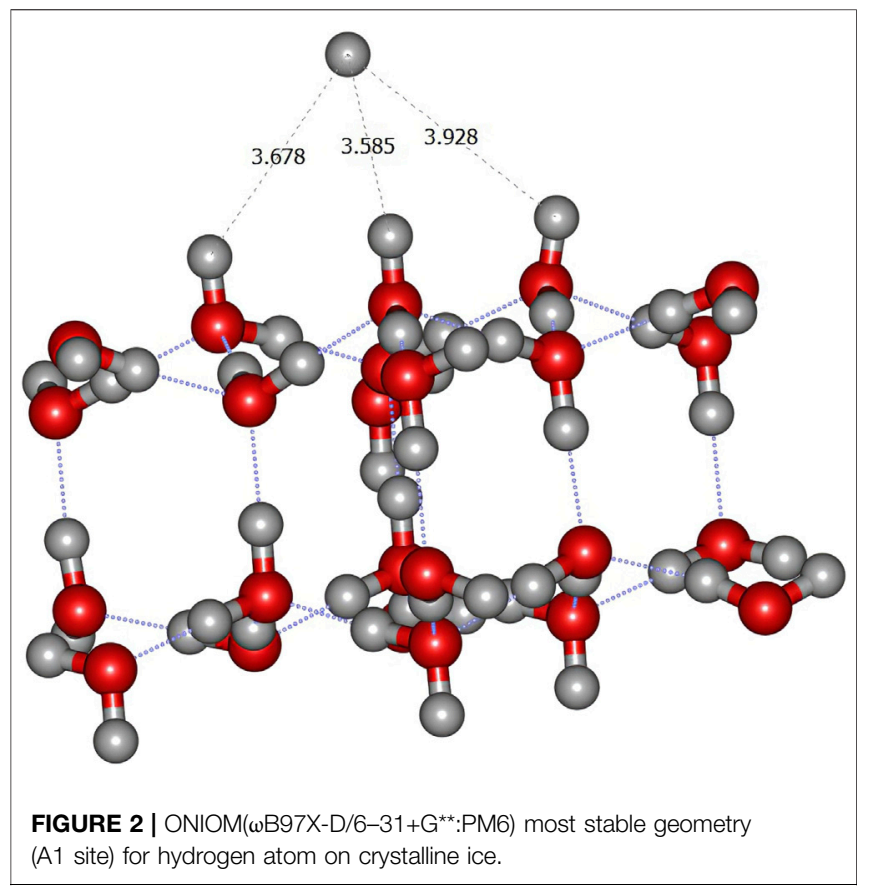

surfaces of interstellar interest. This consists in probing a rich variability of adsorption sites on crystalline or amorphous ices, each of which characterized by a specific binding energy distribution.
A first section presents the details of the methodology and of the performed calculations. Results are then presented. The crystalline ice investigation serves firstly as a benchmark of the model and QM method. Once set, the most accurate combination of methods is then applied on amorphous ices. The obtained values are discussed and compared to published data when available.

\section{METHODS}

As a start, it is important to agree on the definition of the $\mathrm{BE}$ of a species on a substrate. Throughout the paper, BE will be defined as the positive quantity according to the following equation:

$$
B E=\mathrm{E}_{\text {substrate }}+\mathrm{E}_{\text {species }}-\mathrm{E}_{\text {ads }}
$$

Where $E_{a d s}$ is the total calculated energy of the species adsorbed on the surface while $\mathrm{E}_{\text {substrate }}$ and $\mathrm{E}_{\text {species }}$ are obtained separately. Within the goal to describe adsorption on both periodic and amorphous ices, the ONIOM hybrid method has been chosen (Chung et al., 2015) as implemented in the Gaussian 16 code (Frisch et al., 2016). To avoid problems inherent to QM/MM methods, especially in the Mechanical Embedding scheme previously employed (Sameera et al., 2017; Sameera and Maseras, 2018; Sameera et al., 2021), we rather chose a QMHigh:QMLow combination. The QMHigh part was described with Density Functional Theory (DFT). Following previous works from Sameera et al. (Sameera et al., 2017; 
TABLE 1 | Calculated BE's at ONIOM level for atomic species.

\begin{tabular}{|c|c|c|c|c|c|c|c|c|c|}
\hline QMHgh & - & M06-2X & M06-2X & $\omega B 97 X-D$ & $\omega B 97 X-D$ & M06-2X & M06-2X & $\omega B 97 X-D$ & $\omega B 97 X-D$ \\
\hline- & - & $6-31+G^{\star \star}$ & - & - & - & def2-TZVP & - & - & - \\
\hline QMLow & - & PM6 & PM7R8 & PM6 & PM7R8 & PM6 & PM7R8 & PM6 & PM7R8 \\
\hline$H\left({ }^{2} S\right)$ & Minimum & -0.018 & -0.033 & -0.016 & -0.030 & -0.022 & -0.068 & -0.021 & -0.032 \\
\hline- & Maximum & 0.127 & 0.128 & 0.071 & 0.030 & 0.053 & 0.091 & 0.049 & 0.025 \\
\hline- & Average & 0.042 & 0.049 & 0.020 & 0.013 & 0.012 & -0.018 & 0.004 & 0.009 \\
\hline- & $\sigma$ & 0.051 & 0.060 & 0.029 & 0.020 & 0.029 & 0.053 & 0.024 & 0.019 \\
\hline $\mathrm{C}\left({ }^{3} \mathrm{P}\right)$ & Minimum & 0.997 & 1.049 & 0.783 & 0.797 & 1.056 & 1.096 & 0.853 & 0.871 \\
\hline- & Maximum & 1.381 & 1.548 & 1.259 & 1.228 & 1.303 & 1.402 & 1.315 & 1.288 \\
\hline- & Average & 1.130 & 1.220 & 0.974 & 0.997 & 1.163 & 1.268 & 1.045 & 1.083 \\
\hline- & $\sigma$ & 0.144 & 0.176 & 0.158 & 0.156 & 0.094 & 0.116 & 0.160 & 0.156 \\
\hline$N\left({ }^{4} S\right)$ & Minimum & 0.142 & 0.085 & 0.021 & 0.018 & 0.130 & 0.136 & 0.024 & 0.020 \\
\hline- & Maximum & 0.292 & 0.262 & 0.092 & 0.122 & 0.276 & 0.248 & 0.100 & 0.125 \\
\hline- & Average & 0.196 & 0.179 & 0.051 & 0.064 & 0.181 & 0.180 & 0.051 & 0.065 \\
\hline- & $\sigma$ & 0.059 & 0.052 & 0.024 & 0.030 & 0.055 & 0.032 & 0.024 & 0.030 \\
\hline $\mathrm{O}\left({ }^{3} \mathrm{P}\right)$ & Minimum & 0.199 & 0.206 & 0.093 & 0.070 & 0.178 & 0.119 & 0.032 & 0.059 \\
\hline- & Maximum & 0.458 & 0.438 & 0.212 & 0.229 & 0.289 & 0.342 & 0.278 & 0.524 \\
\hline- & Average & 0.263 & 0.299 & 0.149 & 0.175 & 0.212 & 0.224 & 0.150 & 0.207 \\
\hline- & $\sigma$ & 0.087 & 0.078 & 0.036 & 0.049 & 0.036 & 0.084 & 0.078 & 0.140 \\
\hline
\end{tabular}

Sameera and Maseras, 2018), two gaussian atomic basis sets were used, namely Pople's 6-31+G** (Ditchfield et al., 1971; Hehre et al., 1972; Hariharan and Pople, 1973; Clark et al., 1983) and the def2-TZVP (Weigend and Ahlrichs, 2005). The same functionals as in Sameera et al. were also employed: the M06-2X (Zhao and Truhlar, 2008) and the range separated $\omega$ B97X-D (Chai and Head-Gordon, 2008), with the Gaussian 16 default grid size (i.e., ultrafine). For the QMLow part, we used PM6 (Stewart, 2007) and PM7R8 (Throssell and Frisch, 2018; Throssell, 2017) parameters. According to Throssel (Throssell, 2017), the PM7R8 parametrization improves the description of hydrogen bonded systems. For open shell systems, i.e., atoms and radicals, only the high-spin ground state was considered and the unrestricted formalism was employed.

Geometry optimization were carried out in Cartesian coordinates on the QMHigh part, the QMLow zone remaining frozen. Default convergence thresholds on gradients and displacements were used. It should be noticed that no BSSE effect was considered in the present work. For crystalline (Ih) ices, the geometries were obtained from the supplementary material of Sameera et al. (Sameera et al., 2017). They are derived from Karseemeijer et al. (Karssemeijer et al., 2012) and were obtained at the ONIOM(DFT:AMOEBA) level. Thus, two ice models with 4 sites differing by the surface dangling bonds ordering were used. In model A, 162 water molecules are present, with 24 in the QMHigh region. Model B comprises 165 water molecules, the QMHigh zone containing 20 molecules (see Figure 2 in Sameera et al. for a graphical representation). For $\mathrm{OH}, \mathrm{HCO}$, and $\mathrm{CH}_{3}$, the Sameera et al. geometries were used as starting guesses. For the 6 other adsorbates, the initial geometry was generated manually. The harmonic frequencies were obtained at the $\omega \mathrm{B} 97 \mathrm{X}-\mathrm{D} / 6-$ $31+\mathrm{G}^{* *}$ :PM6 level only. The resulting total zero-point vibrational energy correction (ZPE) was then scaled using a factor of 0.9523 taken from the NIST database (https://cccbdb. nist.gov/vsfx.asp). This ZPE correction was then added to the pure electronic BE's obtained will all the different levels of theory employed:

$$
\mathrm{BE}_{0}=\mathrm{BE}_{\mathrm{e}}-\Delta \mathrm{ZPE}\left(\omega B 97 X-D / 6-31+\mathrm{G}^{* *}\right)
$$

For amorphous cases, a different strategy was adopted. An amorphous ice slab was generated by classical molecular dynamics simulations following the procedure detailed in Michoulier et al. (Michoulier et al., 2018), using the TIP4P/ 2005 water model (Abascal and Vega, 2005; Vega and Abascal, 2011). Classical molecular dynamics trajectories have been used to model the adsorption of a single water molecule, described as well with the TIP4P/2005 model, on the generated ice slab at $T=$ $77 \mathrm{~K}$. The goal of these independent trajectories is to sample a variety of adsorption sites that will serve as guess sites for the other adsorbates. The MD simulation details can be found in (Michoulier et al., 2018). The amorphous ice slab contains here 1344 molecules in a simulation box of dimensions: $25.89 \times 29.95$ $\times 90.28 \AA$. The initial position of the adsorbed water molecule is randomly chosen for each trajectory. After a $1 \mathrm{~ns}$ trajectory with a $1 \mathrm{fs}$ timestep at $T=77 \mathrm{~K}$, the final configuration of each trajectory is collected, the conservation of total energy and the convergence of the usual parameters being carefully checked for each trajectory.

The obtained adsorption geometries were cut into 3 different layers. The first one defining the QMHigh part, consists in the absorbed molecule surrounded by a sphere containing about 20 other water molecules. Then, about 120-150 molecules in a slightly larger sphere were selected for the QMLow part and finally the remaining $\sim 1200$ ones were removed. The radii of the spheres were adjusted to obtain approximately the same number of molecules as in the crystalline case. The typical radius of these spheres is about 7-8 $\AA$ for the High-level part and about $12-15 \AA$ for the Low level one. The reduction of the number of water molecules from the slab to a reduced cluster is illustrated in Figure 1. The same ONIOM procedure used for the crystalline phase was applied to these clusters. For reasons explained below, these calculations were performed at the $\omega \mathrm{B} 97 \mathrm{X}-\mathrm{D} / 6-31+\mathrm{G}^{* *}$ : PM6 level only. The other adsorption cases were treated by replacing the water molecule by the corresponding adsorbate 

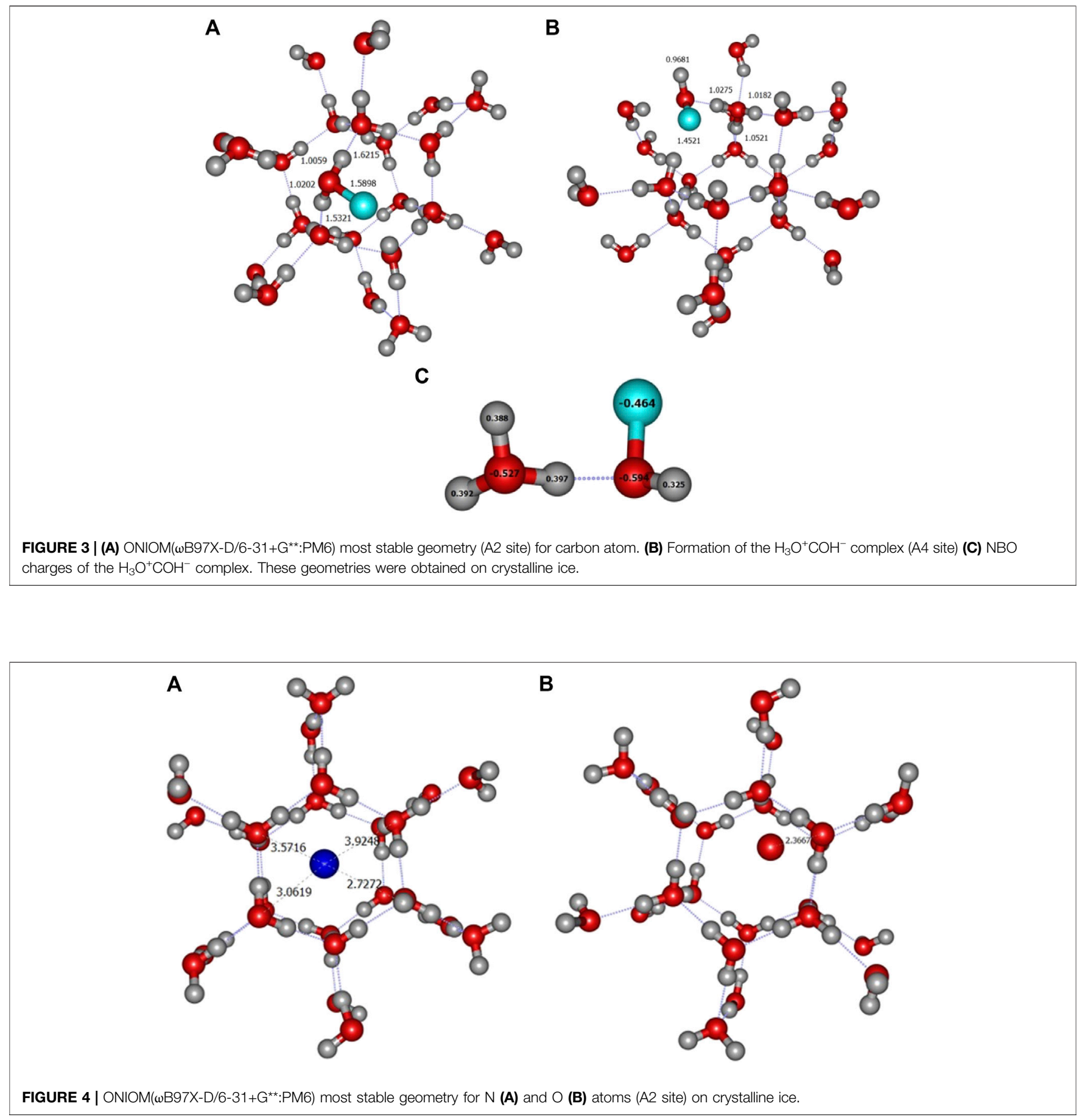

and reoptimizing the geometries. This method proved to give reasonable guess structures for the ONIOM geometry optimization, despite the different nature of the interaction between the various species and the surface. As in the crystalline case, the QMHigh part without the adsorbate (bare ice surface) was optimized separately as well.

The procedure described in the previous paragraphs gives geometry and binding energies at the DFT level. In order to obtain more accurate binding energies, the $\omega \mathrm{B} 97 \mathrm{X}-\mathrm{D} / 6-31+\mathrm{G}^{* *}$ : PM6 QMHigh partial geometries were extracted and used as inputs for a CBS/DLPNO-CCSD(T) (Guo et al., 2018) single point calculation, using the ORCA code (Neese, 2018; Neese et al., 2020). Resolution of the identity (RIJK) was used (Weigend et al., 2009) with the auxiliary basis set from Weigend (Weigend, 2008). For DLPNO-CCSD $(\mathrm{T})$, the normal criteria were used as discussed by Liakos et al. (Liakos et al., 2015). The auxiliary basis set for 
TABLE 2 | Calculated BE's at ONIOM level for diatomic and triatomic systems.

\begin{tabular}{|c|c|c|c|c|c|c|c|c|c|}
\hline QMHigh & - & M06-2X & M06-2X & $\omega b 97 X-D$ & $\omega b 97 X-D$ & M06-2X & M06-2X & $\omega b 97 X-D$ & $\omega b 97 X-D$ \\
\hline- & - & $6-31+G^{*}$ & - & - & - & def2-TZVP & - & - & - \\
\hline QMLow & - & PM6 & PM7R8 & PM6 & PM7R8 & PM6 & PM7R8 & PM6 & PM7R8 \\
\hline $\mathrm{NH}$ & Minimum & 0.267 & 0.318 & 0.146 & 0.124 & 0.256 & 0.368 & 0.148 & 0.109 \\
\hline- & Maximum & 0.840 & 1.114 & 0.790 & 0.864 & 0.855 & 1.154 & 0.819 & 1.082 \\
\hline- & Average & 0.445 & 0.517 & 0.401 & 0.367 & 0.434 & 0.519 & 0.379 & 0.400 \\
\hline- & $\sigma$ & 0.170 & 0.249 & 0.182 & 0.232 & 0.183 & 0.260 & 0.194 & 0.300 \\
\hline $\mathrm{OH}$ & Minimum & 0.426 & 0.421 & 0.274 & 0.444 & 0.408 & 0.401 & 0.446 & 0.343 \\
\hline- & Maximum & 0.672 & 0.747 & 0.670 & 0.718 & 0.713 & 0.711 & 0.764 & 0.698 \\
\hline- & Average & 0.575 & 0.591 & 0.527 & 0.583 & 0.560 & 0.566 & 0.578 & 0.541 \\
\hline- & $\sigma$ & 0.086 & 0.128 & 0.125 & 0.101 & 0.098 & 0.125 & 0.103 & 0.128 \\
\hline $\mathrm{HCO}$ & Minimum & 0.139 & 0.270 & 0.137 & 0.187 & 0.220 & 0.270 & 0.311 & 0.295 \\
\hline - & Maximum & 0.519 & 0.493 & 0.437 & 0.484 & 0.486 & 0.464 & 0.429 & 0.459 \\
\hline- & Average & 0.382 & 0.402 & 0.350 & 0.383 & 0.378 & 0.388 & 0.366 & 0.374 \\
\hline- & $\sigma$ & 0.115 & 0.073 & 0.095 & 0.085 & 0.090 & 0.068 & 0.051 & 0.047 \\
\hline $\mathrm{H}_{2} \mathrm{O}$ & Minimum & 0.607 & 0.627 & 0.558 & 0.610 & 0.572 & 0.592 & 0.553 & 0.585 \\
\hline- & Maximum & 0.857 & 0.857 & 0.834 & 1.117 & 0.831 & 0.830 & 0.823 & 0.808 \\
\hline- & Average & 0.742 & 0.760 & 0.690 & 0.754 & 0.711 & 0.707 & 0.676 & 0.702 \\
\hline- & $\sigma$ & 0.094 & 0.075 & 0.092 & 0.164 & 0.095 & 0.083 & 0.098 & 0.083 \\
\hline
\end{tabular}

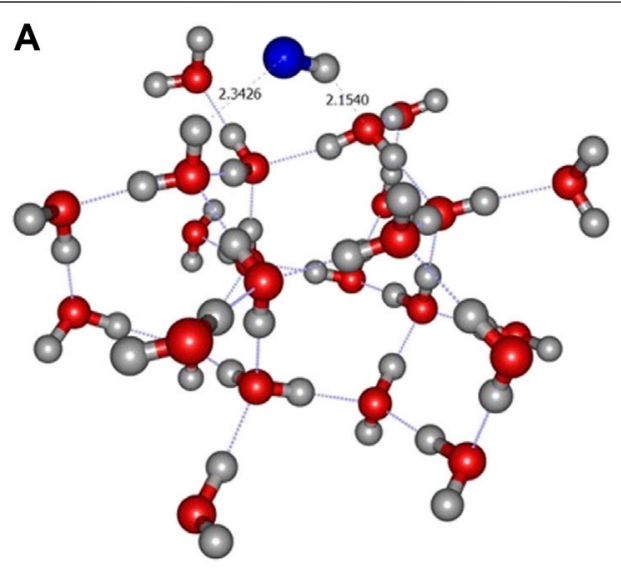

B

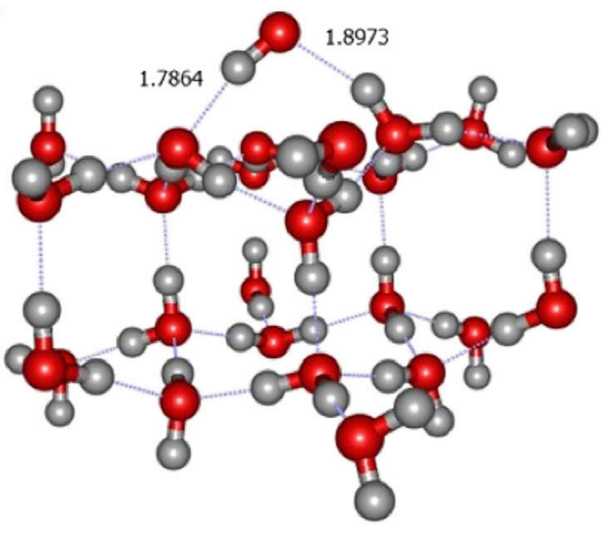

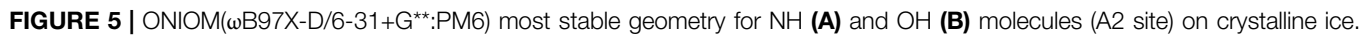

correlation is taken from Hellweg et al. (Hellweg et al., 2007). For Complete Basis Set (CBS) extrapolation, def2-TZVPP and def2QVZPP basis sets were employed (Weigend and Ahlrichs, 2005). The Hartree-Fock and the correlation energies were extrapolated separately as shown in (Neese and Valeev, 2011) with $\alpha=7.880$ for Hartree-Fock and $\beta=2.970$ for correlation. The three (def2-TZVPP, def2-QVZPP and CBS) resulting DLPNO$\operatorname{CCSD}(\mathrm{T})$ energies then replaced the DFT part of the total ONIOM extrapolated energy. The most sophisticated BE can

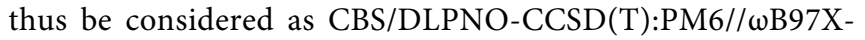
$\mathrm{D} / 6-31+\mathrm{G}^{\star *}: \mathrm{PM} 6$, corrected with the $\omega \mathrm{B} 97 \mathrm{X}-\mathrm{D} / 6-31+\mathrm{G}^{* *}$ : PM6 ZPE. CBS results should also be free of BSSE (Basis Set Superposition Error), but it should also be noted that BSSE effects were calculated to be small by Sameera et al. (Sameera et al., 2017).

In the following, binding energies are expressed in $\mathrm{eV}$. They can be converted in Kelvins using: $1.000 \mathrm{eV}=$ $11604.59 \mathrm{~K}=96.4853 \mathrm{~kJ} / \mathrm{mol}$.

\section{RESULTS AND DISCUSSION}

\section{Crystalline ice}

The study of the crystalline structures was performed to benchmark the various DFT/ONIOM models employed in the present work. Furthermore, the crystal results may be compared with the amorphous ones as well. We have adopted the nomenclature of Sameera et al. (Sameera et al., 2017) who distinguish 8 different adsorption sites (A1, A2, A3, A4, B1, B2, $\mathrm{B} 3$, and B4) depending on the different dangling bonds arrangement at the surface. A detailed comparison with Sameera et al. for the eight sites is given in the supplementary material. In the perspective of studying amorphous ices, we will only report here four characteristic $B E$ 's for each species: the minimum, the maximum, the average values and the standard deviation $\sigma$, respectively. In the following, we present in the figures the geometries corresponding to the most stable configuration (largest $\mathrm{BE}$ ) 

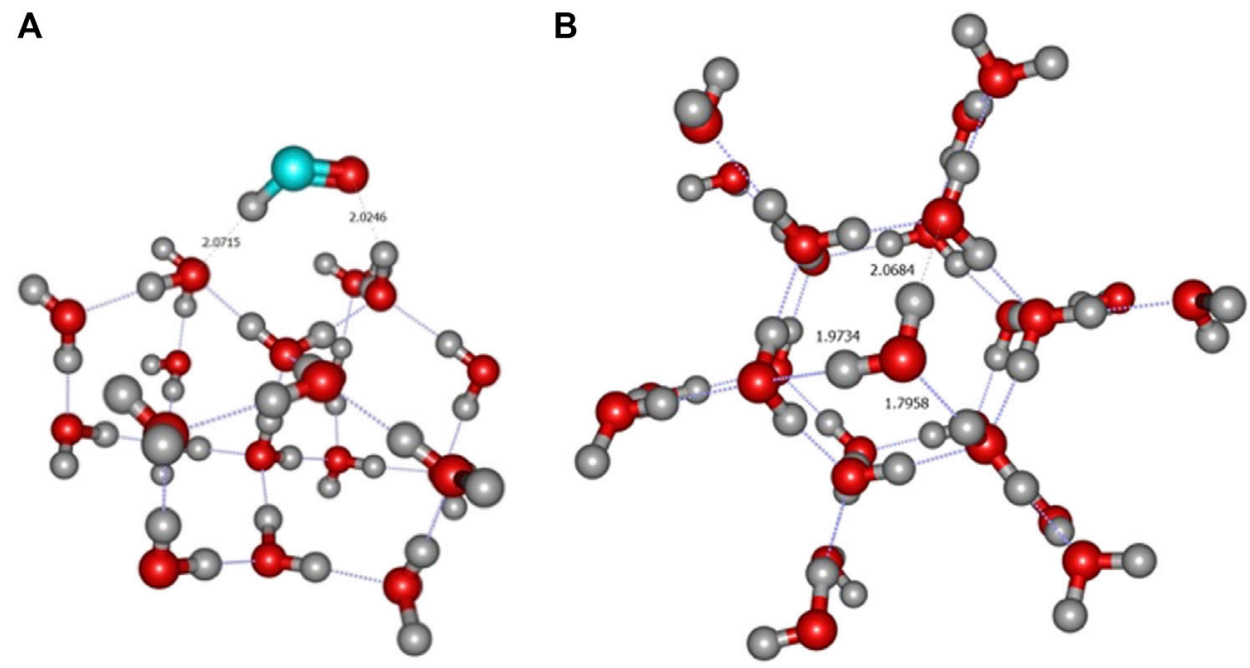

FIGURE 6 | ONIOM( $\omega$ B97X-D/6-31+G**:PM6) most stable geometry for $\mathrm{HCO}$ (B3 site) (A) and $\mathrm{H}_{2} \mathrm{O}$ (A2 site) (B) molecules on crystalline ice.

TABLE 3 | Calculated BE's at ONIOM level for tetratomic systems.

\begin{tabular}{|c|c|c|c|c|c|c|c|c|c|}
\hline QMHigh & - & M06-2X & M06-2X & $\omega b 97 X-D$ & $\omega b 97 X-D$ & M06-2X & M06-2X & $\omega b 97 X-D$ & $\omega b 97 X-D$ \\
\hline - & - & $6-31+G^{*}$ & - & - & - & def2-TZVP & - & - & - \\
\hline QMLow & - & PM6 & PM7R8 & PM6 & PM7R8 & PM6 & PM7R8 & PM6 & PM7R8 \\
\hline $\mathrm{CH}_{3}$ & Minimum & 0.107 & 0.157 & 0.062 & 0.112 & 0.123 & 0.167 & 0.076 & 0.121 \\
\hline- & Maximum & 0.312 & 0.349 & 0.281 & 0.320 & 0.315 & 0.348 & 0.597 & 0.321 \\
\hline- & Average & 0.212 & 0.242 & 0.189 & 0.207 & 0.234 & 0.238 & 0.264 & 0.215 \\
\hline- & $\sigma$ & 0.070 & 0.062 & 0.082 & 0.063 & 0.067 & 0.067 & 0.155 & 0.061 \\
\hline $\mathrm{NH}_{3}$ & Minimum & 0.324 & 0.317 & 0.441 & 0.540 & 0.158 & 0.299 & 0.403 & 0.496 \\
\hline- & Maximum & 0.814 & 1.199 & 0.932 & 1.179 & 0.914 & 1.180 & 0.925 & 1.160 \\
\hline- & Average & 0.571 & 0.649 & 0.660 & 0.708 & 0.579 & 0.642 & 0.658 & 0.666 \\
\hline- & $\sigma$ & 0.211 & 0.288 & 0.163 & 0.219 & 0.231 & 0.259 & 0.173 & 0.221 \\
\hline
\end{tabular}
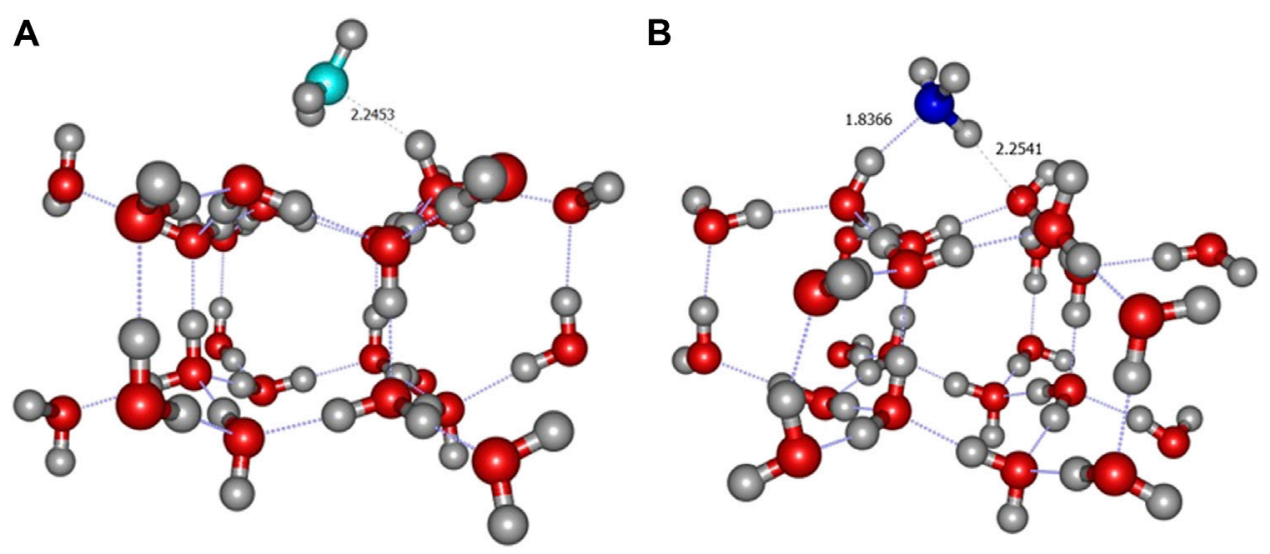

FIGURE 7 | ONIOM( $\omega$ B97X-D/6-31+G**:PM6) most stable geometry for $\mathrm{CH}_{3}$ (A2 site) (A) and $\mathrm{NH}_{3}$ (A3 site) (B) molecules on crystalline ice.

obtained at ONIOM( $\omega$ B97X-D/6-31+G**:PM6) level only. It should be noticed that the most stable geometry depends on the employed functionals, basis sets and semi-empirical methods, as emphasized in the supplementary material. A general trend is that the most stable situation generally corresponds to a A-type site, i.e., above the center of a 
TABLE 4 | Calculated BE's at CBS/DLPNO-CCSD(T):PM6//ONIOM( $\omega$ B97X-D/6-31+G**:PM6) including ZPE for crystalline structures.

\begin{tabular}{|c|c|c|c|c|c|c|c|c|c|c|}
\hline Crystal & $H\left({ }^{2} S\right)$ & $C\left({ }^{3} P\right)$ & $N\left({ }^{4} S\right)$ & $O\left({ }^{3} P\right)$ & NH & OH & HCO & $\mathrm{H}_{2} \mathrm{O}$ & $\mathrm{CH}_{3}$ & $\mathrm{NH}_{3}$ \\
\hline Minimum & -0.017 & 0.474 & -0.001 & 0.008 & 0.078 & 0.139 & 0.133 & 0.343 & 0.006 & 0.232 \\
\hline Maximum & 0.066 & 0.939 & 0.085 & 0.222 & 0.503 & 0.458 & 0.307 & 0.536 & 0.180 & 0.532 \\
\hline Average & 0.013 & 0.714 & 0.031 & 0.084 & 0.234 & 0.341 & 0.215 & 0.422 & 0.074 & 0.385 \\
\hline$\sigma$ & 0.026 & 0.145 & 0.027 & 0.070 & 0.122 & 0.101 & 0.052 & 0.076 & 0.059 & 0.107 \\
\hline Minimum & - & - & - & - & - & $0.20 / 0.15^{a}$ & $0.13 / 0.06^{a}$ & $0.25^{\mathrm{b}}$ & $0.09 / 0.02^{a}$ & - \\
\hline Maximum & - & - & - & -- & - & $0.61 / 0.61^{a}$ & $0.36 / 0.37^{a}$ & $0.68^{\mathrm{b}}$ & $0.27 / 0.30^{a}$ & - \\
\hline Average & - & - & - & - & - & $0.45 / 0.42^{\mathrm{a}}$ & $0.28 / 0.26^{a}$ & $0.51^{\mathrm{b}}$ & $0.19 / 0.16^{a}$ & - \\
\hline$\sigma$ & - & - & - & - & - & $0.15 / 0.14^{a}$ & $0.07 / 0.10^{a}$ & $0.14^{\mathrm{b}}$ & $0.06 / 0.08^{a}$ & - \\
\hline Maximum $^{\mathrm{C}}$ & - & - & - & -- & - & 0.564 & 0.300 & 0.726 & 0.221 & 0.635 \\
\hline Maximum $^{d}$ & - & - & - & & & 0.387 & 0.202 & - & - & 0.366 \\
\hline Maximum $^{\mathrm{e}}$ & - & 1.590 & 0.084 & 0.403 & - & - & - & - & - & - \\
\hline Maximum $^{f}$ & - & - & 0.143 & - & 0.364 & - & - & - & - & - \\
\hline
\end{tabular}

${ }^{a} M 06-2 X / \omega B 97 X-D / 6-31+G^{* *}$ without ZPE from (Sameera et al., 2017).

${ }^{b}$ ONIOM(wB97X-D/def2-TZVP:AMOEBA) without from supplementary material of (Sameera and Maseras, 2018), and -76.437693 a.u. for the $\omega B 97 X-D / d e f 2-T Z V P$ energy of isolated water.

${ }^{c}$ ZPE-corrected periodic B3LYP-D3 and M06-2X/A-VTZ* calculations from (Ferrero et al., 2020a).

${ }^{d} \mathrm{CBS} / \mathrm{CCSD}(\mathrm{T})$ with $2 \mathrm{H}_{2} \mathrm{O}$ calculations from (Ferrero et al., 2020a).

${ }^{e}$ Periodic PBE-D3(BJ)/TZVP without ZPE nor BSSE from (Ferrero et al., 2020b).

${ }^{f}$ ONIOM-like CCSD(T)//M06-2X/TZVP with BSSE from (Ferrero et al., 2020b).

hexagon. There are however some exceptions. For clarity, only the QMHigh part is represented in the figures.

\section{ONIOM DFT Calculations}

We start by examining the results obtained with the 6 combinations of basis sets, functionals and semi-empirical methods.

As displayed in Table 1, the binding energy of the hydrogen atom appears to be very small, in a consistent pattern for all levels of calculations. The slightly negative values correspond to an artefact due to the convergence threshold of the geometry optimization process. It is possible that using tighter convergence criteria on the geometry optimization process would solve this issue. However, the Potential Energy Surface (PES) is indeed very flat, with the $\mathrm{H}$ atom roaming almost freely above the surface (see e.g., Figure 2). Hence the convergence of the SCF process can be very slow, even using the expensive quadratic convergence algorithm (Schlegel and McDouall, 1991) and the computational cost is quite high.

The carbon atom case is also specific since all calculations, with all 6 levels of theory, give a rather large $\mathrm{BE}$, above $1 \mathrm{eV}$ (Table 1). The length of the $\mathrm{C}-\mathrm{O}$ bond, is about $1.6 \AA$ (see Figure 3A). This is in agreement with recent theoretical work on crystalline (Ferrero et al., 2020b) and amorphous (Shimonishi et al., 2018) ices who conclude on a chemisorption rather than physisorption. Indeed, in the triplet state of gas phase $\mathrm{OCH}_{2}$, the molecule is slightly pyamidalized and the $\mathrm{C}-\mathrm{O}$ bond is elongated to a larger value of $\sim 1.88 \AA$ (Hickson et al., 2016). Ferrero et al. also find a transfer of a hydrogen atom to a nearby water molecule to form a $\mathrm{COH}$ radical. Furthermore, a Mulliken population analysis shows that this radical has a negative charge. Our own calculations show a slightly different picture: a Wiberg bond index analysis (Wiberg, 1968) confirms a weak covalent bond between $\mathrm{C}$ and $\mathrm{O}$ (0.73 for C for A2 site). Furthermore, a NBO population analysis (Weinhold and Carpenter, 1988), shows that the $\mathrm{COH}_{2}$ remains essentially neutral. The only exception is the
A4 site, for which, at all levels of calculation, the adsorption of the carbon atom leads to the loss of a $\mathrm{H}$ atom from the attacked $\mathrm{H}_{2} \mathrm{O}$ (Figure 3B). The NBO analysis this time confirms that $\mathrm{COH}$ fragment carries a total charge of -0.733 while the $\mathrm{H}_{3} \mathrm{O}$ part has a +0.650 positive charge (Figure $3 \mathrm{C}$ ). Thus, this geometry corresponds to the formation of a $\mathrm{H}_{3} \mathrm{O}^{+}-\mathrm{COH}^{-}$complex, very much like in a solvated situation and in agreement with Ferrero et al. (Ferrero et al., 2020b).

On the other hand, the last two studied atoms, namely $\mathrm{N}$ and $\mathrm{O}$ in their respective ground state, have weak interaction energies, characteristic of physisorption (Table 1). The most stable geometries are displayed in Figures 4A,B, respectively, Table 1 also shows a remarkably large difference between the M06-2X and $\omega B$ 97X-D, independent of the basis sets and semiempirical methods used. The M06-2X are about twice larger, despite the fact that the geometries are very similar for all sites.

Table 2 summarizes the results for the 4 studied diatomic and triatomic molecules $\left(\mathrm{NH}, \mathrm{OH}, \mathrm{HCO}\right.$ and $\left.\mathrm{H}_{2} \mathrm{O}\right)$. The corresponding lowest lying equilibrium geometries are displayed in Figures 5, 6. The maximum $\mathrm{BE}$ of $\mathrm{NH}$ is about $50 \%$ larger than for $\mathrm{OH}$, which is typical of hydrogen bonding (David et al., 2017). For $\mathrm{OH}$, the maximum BE, around $0.7 \mathrm{eV}$ depending on the level of calculation, is typical of the formation of two hydrogen bonds, in agreement with previous theoretical works (Du et al., 2006; Guedes et al., 2003), as shown in Figure 5B.

The situation is very similar in the $\mathrm{HCO}$ case, which creates two hydrogen bonds as well (Figure 6A) although the $\mathrm{BE}$ is slightly lower (about $0.5 \mathrm{eV}$ ). For $\mathrm{H}_{2} \mathrm{O}$, the rather high value (about $0.6 \mathrm{eV}$ according to Table 2), is consistent with the building of three hydrogen bonds in the most favorable conformation shown in Figure 6B.

The $\mathrm{BE}$ of $\mathrm{CH}_{3}$ is consistently predicted to be around $0.3 \mathrm{eV}$ (Table 3) and this radical is only bound by a weak interaction as can be seen in Figure 7A. In contrast, for ammonia, the calculated binding energies appear to be rather high, between 0.8 and $1.2 \mathrm{eV}$ 


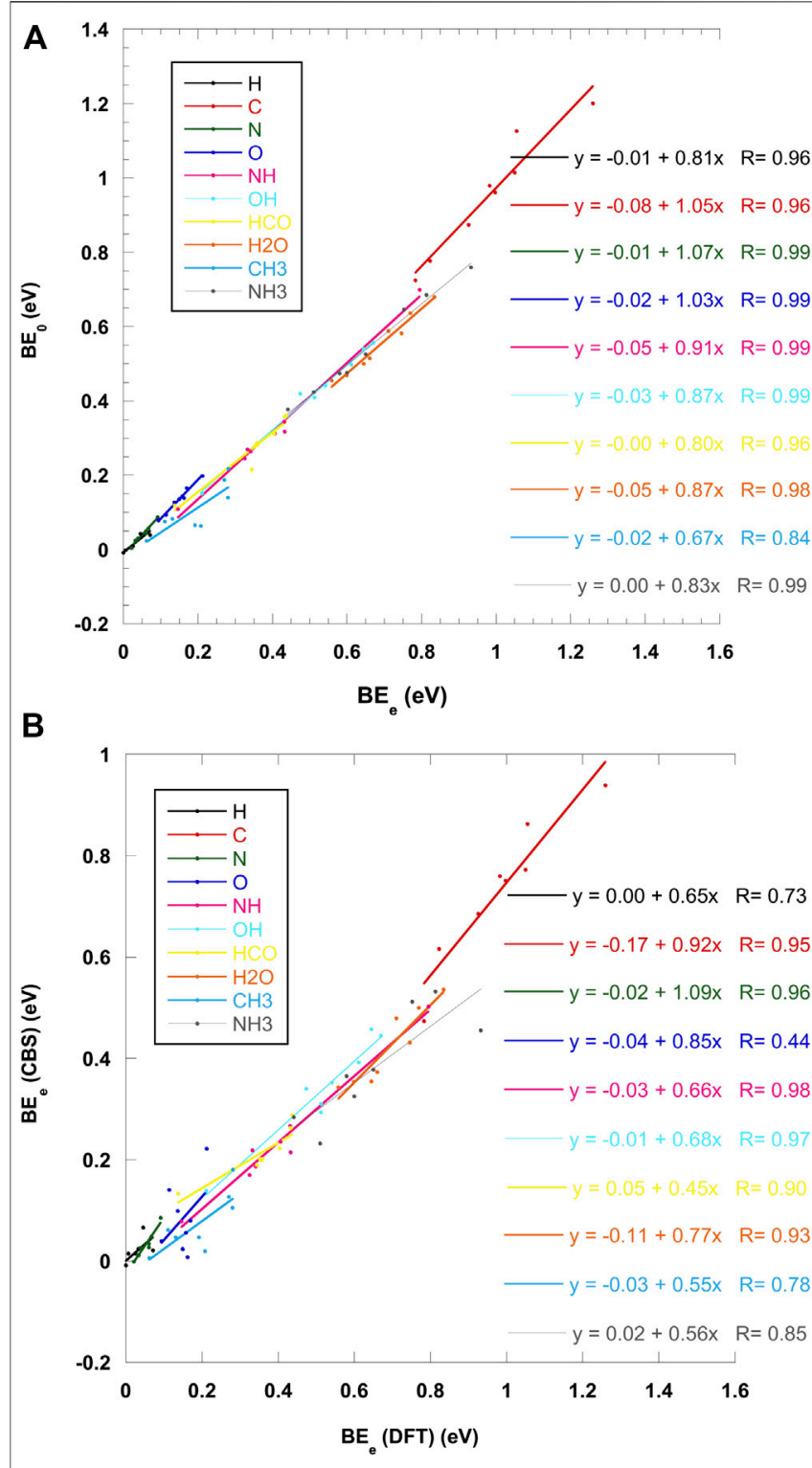

FIGURE 8 | Effect of scaled ZPE on ONIOM( $\omega$ B97X-D/6-31+G**:PM6) BE's for crystalline ices (A) Effect of CBS single point on ZPE-corrected BE's for crystalline ices (B).

depending on the level of theory (Table 3). This is correlated to the existence of two hydrogen bonds between $\mathrm{NH}_{3}$ and two water molecules as displayed in Figure $\mathbf{7 B}$. This is consistent with the intermolecular energy obtained for the isolated $\mathrm{NH}_{3}-\mathrm{H}_{2} \mathrm{O}$ dimer $(0.473 \mathrm{eV})$ (Wakelam et al., 2017).

From the inspection of the average energies given in Tables 1-3, and the fact that all methods happened to give very similar geometries, we have selected the $\omega \mathrm{B} 97 \mathrm{X}-\mathrm{D} / 6-31+\mathrm{G}^{* *}: \mathrm{PM} 6$ combination to obtain the ZPE correction and apply the single point CBS correction. Indeed, the M06-2X gives too large values for $\mathrm{N}$ and $\mathrm{O}$ atoms compared to $\omega \mathrm{B} 97 \mathrm{X}-\mathrm{D}$. This latter functional, being range-separated describes more accurately long-range interactions. Basis sets effects seem to be small and the $6-31+\mathrm{G}^{* *}$ one is much less demanding. Finally, PM6 was selected over PM7R8 since this latter method, although available in the Gaussian 16 code and detailed in the PhD work of Throssell (Throssell, 2017), does not seem to have been published yet.

\section{CBS Calculations}

Table 4 shows the summary of the results obtained for crystalline ices at our best level of calculations, i.e., CBS/DLPNO-CCSD(T): $\mathrm{PM} 6 / / \mathrm{ONIOM}\left(\omega \mathrm{B} 97 \mathrm{X}-\mathrm{D} / 6-31+\mathrm{G}^{* *}: \mathrm{PM} 6\right)$ including scaled ZPE correction calculated at the $\operatorname{ONIOM}\left(\omega \mathrm{B} 97 \mathrm{X}-\mathrm{D} / 6-31+\mathrm{G}^{\star *}\right.$ : PM6) level. A graphical representation is shown in Figure 8. The impact of ZPE on BE's is shown in Figure 8A Except for the case of $\mathrm{CH}_{3}$, a linear fit gives a correlation coefficient larger than 0.96. As expected, ZPE effects are larger for polyatomic molecules. For C, N, and O, the $\mathrm{BE}$ tends to increase by 3-7\%. For molecules, the ZPE lowers the BE's, the drop varying from 10 to $20 \%$ with the notable exception of $\mathrm{CH}_{3}$ where it reaches $33 \%$. Recently, Ferrero et al. (Ferrero et al., 2020a) have obtained the relation $\mathrm{BE} 0=0.854 \mathrm{BEe}$ using a fragment approach to calculate ZPE effects on crystalline ice. Their set of species does not include any atomic system but contains larger molecules, leading to much larger ZPE's.

In addition, single point $\mathrm{CBS} / \mathrm{DLPNO}-\mathrm{CCSD}(\mathrm{T})$ calculations have also some effect on the BE's, as illustrated in Figure 8B. The correction is more erratic, with a bad $(<0.9)$ correlation coefficient for $\mathrm{H}, \mathrm{O}, \mathrm{CH}_{3}$, and $\mathrm{NH}_{3}$. Except for the nitrogen atom where CBS tends to augment the BE by $9 \%$, the other values are lowered, sometimes considerably (up to 55\% for HCO for example). More details on ZPE and CCSD(T) corrections for each of the eight sites can be found in the supplementary material file.

Table 4 also compares when available (i.e., $\mathrm{OH}, \mathrm{HCO}, \mathrm{H}_{2} \mathrm{O}$, and $\mathrm{CH}_{3}$ ) the present results to the single point DFT calculations on Mechanical Embedding (ME) ONIOM(DFT/AMOEBA) geometries of Sameera et al. (Sameera et al., 2017; Sameera and Maseras, 2018). It should be noticed that since these calculations do not include any ZPE, the BE's are larger than the present results. In addition, the maximum values given in Table 4 correspond to the most stable configuration on a perfect crystalline ice. Our results can also be compared to the recent values obtained by Ferrero et al. (Ferrero et al., 2020a; Ferrero et al., 2020b) using periodic DFT methods and given at the bottom of Table 5. Except for $\mathrm{N}$ atom, our values for $\mathrm{C}$ and $\mathrm{O}$ are much lower than the periodic PBE-D3(BJ)/TZVP of Ferrero et al. (Ferrero et al., 2020b). The recent study of Martinez-Bachs et al. (Ferrero et al., 2020b) performed in an ONIOM-like CCSD(T)// M06-2X/TZVP scheme provides a rather high value for $\mathrm{N}$ $(0.143 \mathrm{eV})$ compared to our maximum $(0.083 \mathrm{eV})$. On the other hand, their value for $\mathrm{NH}(0.364 \mathrm{eV})$ is lower than our maximum result equal to $0.503 \mathrm{eV}$. Except for the $\mathrm{HCO}$ case where the two values coincide, CBS values are also lower than the CCSD(T) ones obtained by Ferrero et al. (Ferrero et al., 2020a), but it should be kept in mind that these authors limited the calculation to only two water molecules in addition to the adsorbate. 


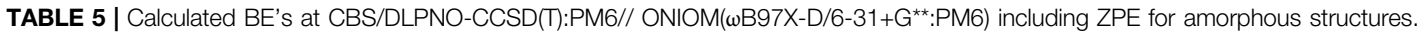

\begin{tabular}{|c|c|c|c|c|c|c|c|c|c|c|}
\hline ASW & $H\left({ }^{2} S\right)$ & $\mathrm{C}\left({ }^{3} \mathrm{P}\right)^{\mathrm{a}}$ & $N\left({ }^{4} S\right)$ & $O\left({ }^{3} P\right)$ & NH & $\mathrm{OH}$ & $\mathrm{HCO}$ & $\mathrm{H}_{2} \mathrm{O}$ & $\mathrm{CH}_{3}$ & $\mathbf{N H}_{3}$ \\
\hline Minimum & -0.005 & 0.612 & 0.027 & 0.015 & 0.016 & 0.253 & -0.032 & 0.359 & 0.080 & 0.208 \\
\hline Maximum & 0.058 & 1.118 & 0.093 & 0.126 & 0.314 & 0.769 & 0.246 & 0.564 & 0.167 & 0.530 \\
\hline Average & 0.010 & 0.816 & 0.050 & 0.063 & 0.210 & 0.448 & 0.156 & 0.467 & 0.114 & 0.371 \\
\hline$\sigma$ & 0.020 & 0.132 & 0.020 & 0.032 & 0.097 & 0.167 & 0.099 & 0.067 & 0.036 & 0.120 \\
\hline Minimum $^{b}$ & - & - & - & - & - & 0.134 & 0.113 & 0.311 & 0.096 & 0.372 \\
\hline Maximumb & - & - & - & - & - & 0.459 & 0.265 & 0.527 & 0.143 & 0.650 \\
\hline Dimer $^{\mathrm{C}}$ & 0.034 & 0.862 & 0.103 & $0.146 / 0.190$ & 0.241 & $\begin{array}{l}0.457 / \\
0.284\end{array}$ & 0.198 & 0.396 & 0.215 & 0.483 \\
\hline Dimer $^{d}$ & 0.059 & - & 0.078 & $0.155 / 0.129$ & 0.207 & 0.414 & 0.181 & 0.336 & 0.129 & 0.474 \\
\hline 4/6 $\mathrm{H}_{2} \mathrm{O}$ cluster $^{\mathrm{e}}$ & 0.011 & 0.057 & $0.023 / 0.053$ & 0.086/0.057 & 0.168 & 0.274 & 0.160 & 0.230/0.359 & 0.114 & $\begin{array}{l}0.330 / \\
0.445\end{array}$ \\
\hline $20 \mathrm{H}_{2} \mathrm{O}$ cluster $^{f}$ & - & $\begin{array}{c}1.215 \pm \\
0.036\end{array}$ & $\begin{array}{c}0.034 \pm \\
0.003\end{array}$ & $\begin{array}{c}0.124 \pm \\
0.014\end{array}$ & - & - & - & - & - & - \\
\hline $500 \mathrm{H}_{2} \mathrm{O}$ cluster 9 & - & - & $\begin{array}{c}0.030 \pm \\
0.013\end{array}$ & - & - & - & - & - & - & - \\
\hline $\begin{array}{l}\text { 18/33 } \mathrm{H}_{2} \mathrm{O} \\
\text { cluster }^{\mathrm{h}}\end{array}$ & - & - & - & - & - & - & - & $\begin{array}{c}0.186 / 0.175 / \\
0.230\end{array}$ & $\begin{array}{c}0.074 / 0.052 / \\
0.098\end{array}$ & - \\
\hline Classical MD ${ }^{i}$ & $\begin{array}{c}0.056 \pm \\
0.09\end{array}$ & - & - & - & - & - & & - & - & - \\
\hline ONIOM & - & - & - & - & - & $0.06-0.74$ & - & - & - & - \\
\hline
\end{tabular}

${ }^{a}$ Excludes the $\mathrm{COH}^{-} \mathrm{H}_{3} \mathrm{O}^{+}$case.

${ }^{b} Z P E$ corrected B3LYP-D3 and M06-2X/A-VTZ*//HF-3c from (Ferrero et al., 2020b).

${ }^{c}$ M06-2X/aug-cc-pVTZ without ZPE nor BSSE from Wakelam et al. (Wakelam et al., 2017).

${ }^{d}$ MP2/aug-cc-pVTZ without ZPE nor BSSE from Wakelam et al. (Wakelam et al., 2017).

${ }^{e} M P 2 / a u g-c c-p V D Z$ from Das et al. (Das et al., 2018).

${ }^{f} \omega b 97 X-D / 6-311+G(d, p)$ from Shimonishi et al. (Shimonishi et al., 2018).

${ }^{g}$ Obtained using Molecular Dynamics with Machine Learning Potential from Molpeceres et al. (Molpeceres et al., 2020).

${ }^{h}$ BHLYP/6-31+G (d,p) with D3 and BSSE corrections from E nrique-Romero et al. (Enrique-Romero et al., 2019).

${ }^{i}$ From (Al-Halabi and Van Dishoeck, 2007).

jONIOM(wb97X-D/def2 (TZVP:AMBER) average on both crystalline and amorphous ice from (Miyazaki et al., 2020). Average at 0.37 eV.

\section{Amorphous ice}

Table 5 shows the summary of the results obtained for amorphous ices at the CBS/DLPNO-CCSD(T):PM6// ONIOM( $\omega$ B97X-D/6-31+G**:PM6) including scaled ZPE correction. This table also displays when available previous theoretical determinations of the BE's. A graphical comparison with the crystalline case can be seen in Figure 11. Figure 9A compares the corrected $\mathrm{BE}_{0}$ to the uncorrected $\mathrm{BE}_{\mathrm{e}} \mathrm{DFT}$ results. If we except the special case of hydrogen atom, the correlation coefficient is always larger than 0.97 . For the three other atoms, the ZPE correction is in fact very small (a few \%). For the molecules, the correction is between 11 and $20 \%$, except for $\mathrm{CH}_{3}$ where it is $32 \%$ as in the crystalline case. Figure $9 \mathrm{~B}$ plots the comparison between the CBS/DLPNO-CCSD(T) and the DFT calculations. The dispersion of the values is much larger than in the crystalline case, except for the carbon atom where a covalent bond is created. However, it is clear that the CBS results are considerably lower than the DFT ones.

It should be noticed that, as in the $\mathrm{A} 4$ site of crystalline ice, one of the amorphous clusters produced a $\mathrm{H}_{3} \mathrm{O}^{+} \mathrm{COH}^{-}$complex when approached by a carbon atom in its triplet ground state (see Figure 10A). The charges of this product were confirmed via a $\mathrm{NBO}$ analysis (Figure 10B). The $\mathrm{H}_{3} \mathrm{O}^{+} \mathrm{COH}^{-}$structure lies about $1.1 \mathrm{eV}$ below the dissociated case, which is the largest calculated value. For the seven other amorphous structures, the final product was the pyramidal triplet state of the $\mathrm{COH}_{2}$ complex already found in crystalline ice.
According to Table 5, our best estimate for the binding energy of $\mathrm{H}$ is $0.010 \pm 0.020 \mathrm{eV}$. However, the maximum value is $0.058 \mathrm{eV}$. They are close to the crystalline case (Table 4). Previous theoretical works, either using quantum methods on clusters [(Wakelam et al., 2017; Das et al., 2018)] or classical calculations (Al-Halabi and Van Dishoeck, 2007) also give very dispersed values. One can only conclude that while on average the $\mathrm{BE}$ is very small for $\mathrm{H}$, some local configuration of ASW may lead to larger values as large as $0.058 \mathrm{eV}$.

Concerning the carbon atom, the low value obtained by Das et al. (Das et al., 2018), i.e., only $0.057 \mathrm{eV}$ is easily explained by the fact that the $\mathrm{C}$ atom is weakly bound to a hydrogen atom instead of an oxygen [see Figure 3A in supplementary material in (Das et al., 2018)]. Our average value of $0.816 \pm 0.132 \mathrm{eV}$ is $33 \%$ lower than average of $1.215 \pm 0.036 \mathrm{eV}$ obtained at the $\omega \mathrm{b} 97 \mathrm{X}-\mathrm{D} / 6-311$ $+\mathrm{G}(\mathrm{d}, p)$ level by Shimonishi et al. (Shimonishi et al., 2018). The differences come from the lack of Low level layer, the absence of ZPE and CBS single point correction which impact the BE's. Their smaller uncertainty is possibly due to a different method to select the adsorption trial geometries and average the largest BE's.

The binding energy of nitrogen atom on ASW has been measured experimentally by Minissale et al. (Minissale et al., 2016) to be $0.062_{-0.007}^{+0.014} \mathrm{eV}$. This is in reasonable agreement with our CBS result of $0.050 \pm 0.020 \mathrm{eV}$ but somewhat larger than the recent values of $0.034 \pm 0.003 \mathrm{eV}$ (Shimonishi et al., 2018) and $0.030 \pm 0.013 \mathrm{eV}$ (Molpeceres et al., 2020). 


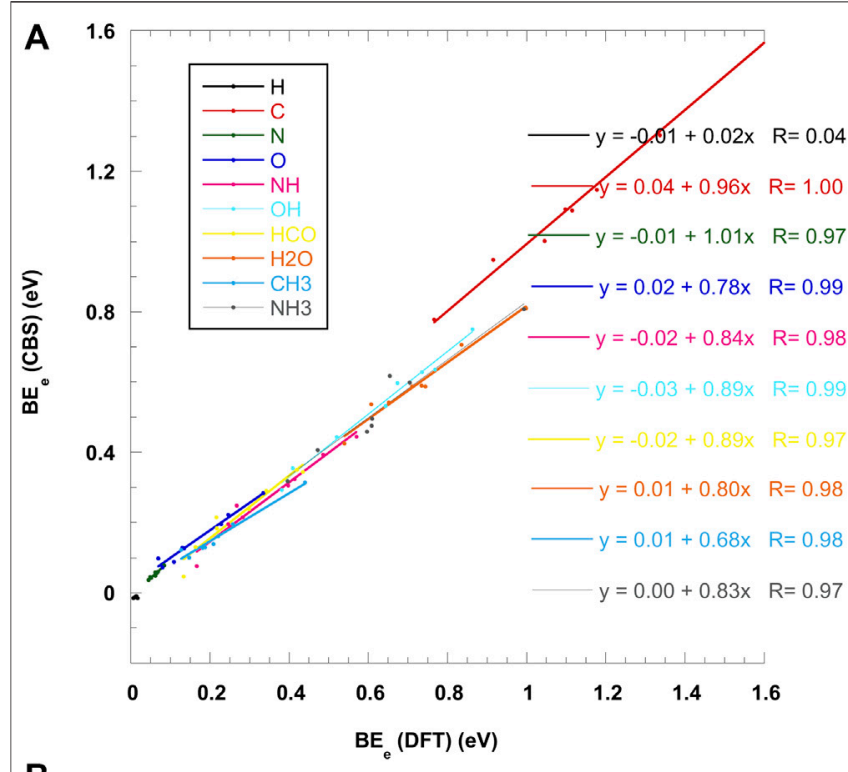

B

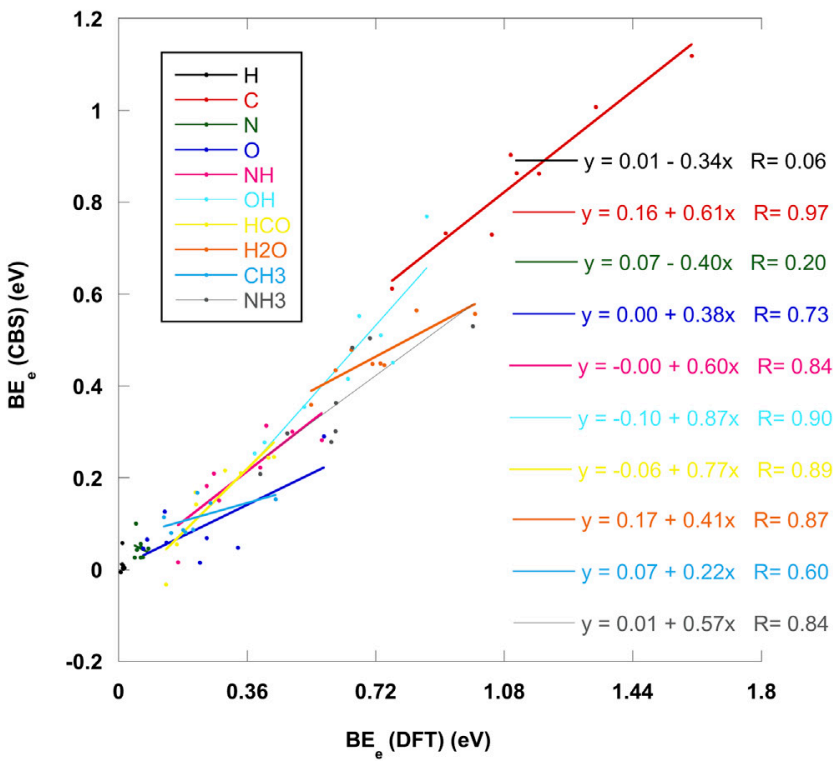

FIGURE 9 | Effect of scaled ZPE on ONIOM( $\omega$ B97X-D/6-31+G*:PM6) BE's for amorphous ices (A) Effect of CBS single point on ZPE-corrected BE's for amorphous ices (B).

Minissale et al. also measured the $\mathrm{BE}$ for oxygen atom: $0.122_{-0.014}^{+0.025} \mathrm{eV}$. Other experimental values include $0.143 \pm$ $0.005 \mathrm{eV}$ from porous ASW (He et al., 2015) and $0.152 \pm$ $0.020 \mathrm{eV}$ on silicate surfaces (He et al., 2014). Our best estimate for the average value (Table 5) is $0.090 \mathrm{eV}$ with a standard deviation of $0.032 \mathrm{eV}$. This is close to the other theoretical values of $0.124 \pm 0.014 \mathrm{eV}$ obtained by Shimonishi et al. (Shimonishi et al., 2018) on a 20 water molecules water cluster. Thus, most previous works are consistent with our maximum value equal to $0.126 \mathrm{eV}$.

To the best of our knowledge, there is no experimental determination of the $\mathrm{BE}$ of the $\mathrm{NH}$ radical on ice surfaces. As shown in Table 5, only calculations on water clusters are available. Our best estimate of $0.210 \pm 0.097 \mathrm{eV}$ is slightly higher than the $0.168 \mathrm{eV}$ value obtained on a water tetramer by Das et al. (Das et al., 2018) but close to the dimer result $(0.207 \mathrm{eV})$ from Wakelam et al. (Wakelam et al., 2017).

Similarly, for the $\mathrm{OH}$ radical, no experimental value has been published yet. For example, for $\mathrm{OH}$, Garrod and Herbst (Garrod and Herbst, 2006) assumed that it is half the value of $\mathrm{H}_{2} \mathrm{O}$, i.e., $0.246 \mathrm{eV}$. Dulieu et al. (Dulieu et al., 2013) obtained $0.396 \mathrm{eV}$ but on silicate dust surfaces. Therefore, as for other molecules, only theoretical values are available, most of them obtained with water clusters (Table 5). The only exception is the recent work by Ferrero et al. (Ferrero et al., 2020b), where the BE of $\mathrm{OH}$ is calculated to be between 0.134 and $0.459 \mathrm{eV}$ (on 5 amorphous structures). Our maximum value of $0.769 \mathrm{eV}$ is higher, and could be explained by the existence of three hydrogen bonds between $\mathrm{OH}$ and the neighboring water molecules. Our average best estimate is thus $0.448 \pm 0.167 \mathrm{eV}$.

As for HCO, again no experimental value is available. The value of Garrod and Herbst (Garrod and Herbst, 2006) of $0.138 \mathrm{eV}$ was deduced by addition and subtraction of energies from other molecules. In Table 5, our slightly negative value is also due to the large CBS/DLPNO-CCSD(T) correction, the first positive value being in fact $0.055 \mathrm{eV}$, which is lower than the minimum value of Ferrero et al. at $0.116 \mathrm{eV}$. On the other hand, our maximum values are very similar: $0.246 \mathrm{eV}$ in the present work and $0.265 \mathrm{eV}$ in Ferrero et al.

In contrast to most other species studied in the present work, the adsorption of water on ice has been the subject of numerous experimental works [see Table 1 in (Yocum et al., 2019) for a recent review]. Sandford and Allamandola (Sandford and Allamandola, 1988) proposed $0.415 \pm 0.001$ and $0.437 \pm$ $0.001 \mathrm{eV}$, Fraser et al. (Fraser et al., 2001) $0.497 \pm 0.005 \mathrm{eV}$, Haynes et al. (Haynes et al., 1992) $0.516 \pm 0.009 \mathrm{eV}$, Bolina et al. (Bolina et al., 2005) $0.414 \pm 0.008 \mathrm{eV}$, Ulbricht et al. (Ulbricht et al., 2006) $0.477 \pm 0.031 \mathrm{eV}$. Although the experiments differ in the details (for example the substrate), they agree to a value between $0.4-0.5 \mathrm{eV}$. This is in good agreement with our best estimate of $0.467 \pm 0.067 \mathrm{eV}$ (Table 5).

$\mathrm{CH}_{3}$ being a radical as well, experimental determinations seem inexistent. A recent DFT study by Enrique-Romero et al. (Enrique-Romero et al., 2019) on water clusters give values below $0.1 \mathrm{eV}$ (Table 5). Our own results are slightly higher with $0.114 \pm 0.036 \mathrm{eV}$. Our minimum and maximum values are also similar to those of Ferrero et al. (Ferrero et al., 2020b).

Finally, for $\mathrm{NH}_{3}$, the recommended value of $0.477 \mathrm{eV}$ (Hama and Watanabe, 2013) is estimated from the experimental TPD experiments from Collings et al. (Collings et al., 2004). As can be seen in Table 4, this value is close to the water clusters calculation [(Wakelam et al., 2017; Das et al., 2018)] and lies within the interval of our best estimate: $0.371 \pm 0.120 \mathrm{eV}$. The DFT//HF-3c results of Ferrero et al. (Ferrero et al., 2020b), favor larger values between 0.372 and $0.650 \mathrm{eV}$.

Figure 11. A gives finally a graphical representation of the CBS BEs obtained on the 16 adsorption sites, 8 crystalline and 8 amorphous structures. It shows that BE's can vary significantly 

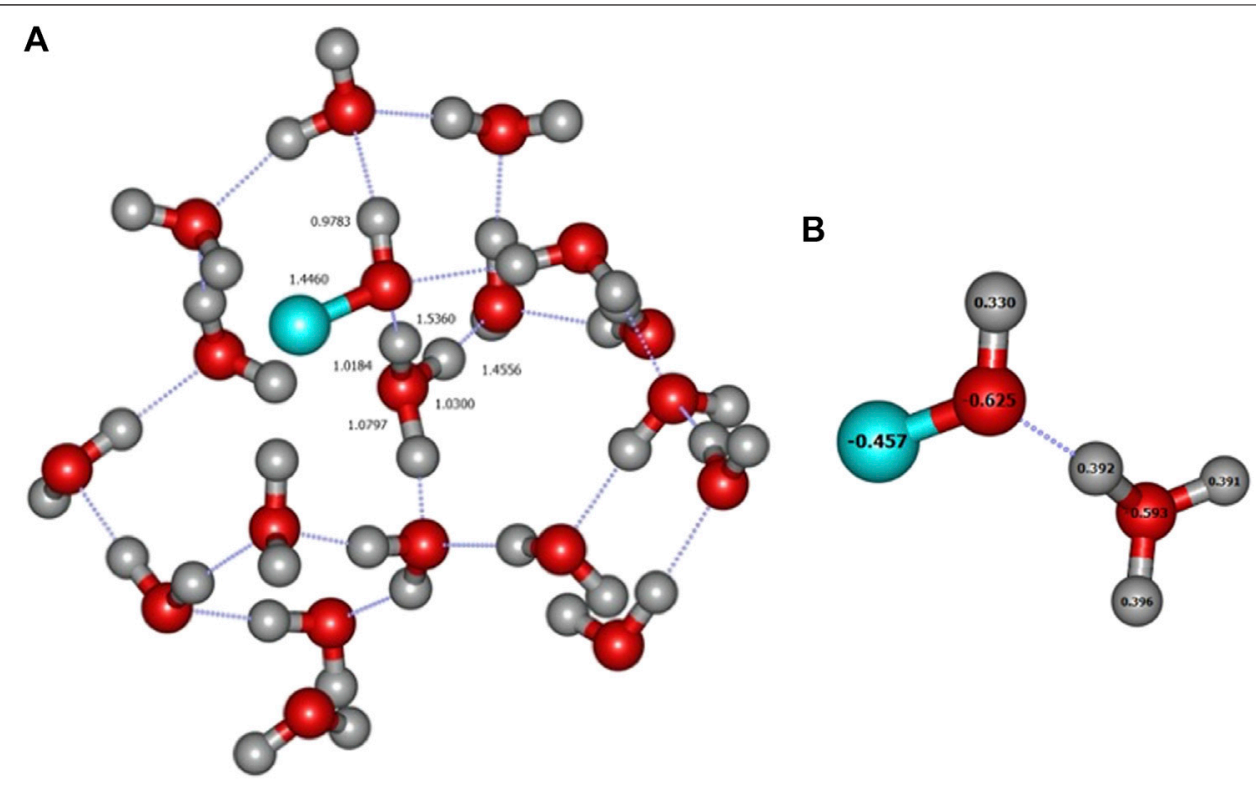

FIGURE 10 | (A) Formation of the $\mathrm{H}_{3} \mathrm{O}^{+} \mathrm{COH}^{-}$complex in an amorphous environment. Distances are given in Angströms. (B) NBO charges from ONIOM( $\omega$ B97X-D/ $\left.6-31+G^{* *}: P M 6\right)$ calculation.

for a given adsorbate or a given substrate, depending strongly on the local environment. Excepting the reactive carbon atom case, the highest value should correspond to the lowest lying equilibrium geometry. However, in most cases, several configurations have very similar BE's, both for crystalline and amorphous cases, these values being indicative of a configuration that may exist on any ice sample. It is worth mentioning that the statistics could be improved by considering a larger number of initial configurations, but it has to be reminded that for each geometry, a full QM/MM optimization and single point CBS/DLPNO-CCSD(T) correction are carried out, these calculations being expensive, more especially when convergence is difficult to achieve. Figure 11B compares the average BE's and their standard deviation for both surfaces. In most cases, the amorphous average is above the crystalline value, but the trend is not universal and does not allow to conclude without ambiguity. It should be recalled that the considered amorphous ice surface is non-porous, addition of pores or cavities could lead to more marked differences with respect to the crystalline face. However, such a surface would be difficult to handle with the proposed methodology because of the large cluster size it would require. The comparison of our calculated BE's with the values that have been reported in UMIST (McElroy et al., 2013) or KIDA (Wakelam et al., 2012) databases indicate that the values appearing in the databases are for most cases contained in the BE's range we obtain on both surfaces. For $\mathrm{OH}, \mathrm{HCO}$, and $\mathrm{C}$, the data implemented in KIDA have a better agreement with our theoretical values than the UMIST ones. This comparison further supports our methodology that can be used to provide BE's for species remaining undocumented, such as radicals or atoms or even larger molecules, thus improving the chemical networks implemented in the astrochemical models.

\section{CONCLUSION}

In this work we have used a first principles methodology tailored to study adsorption processes of small atoms and molecules on both crystalline and amorphous ices, represented by a cluster of about 150 water molecules. A full quantum ONIOM QMHigh/QMLow scheme was employed, using semi-empirical methods for the surrounding water molecules representative of the ice sample.

Several combinations of functionals, basis sets and semiempirical parametrizations were tested on the different possible adsorption sites of a perfect hexagonal ice. The ONIOM $\left(\omega \mathrm{B} 97 \mathrm{X}-\mathrm{D} / 6-31+\mathrm{G}^{* *}: \mathrm{PM} 6\right)$ was chosen as a good compromise between cost and accuracy. Despite the fact that the $\omega \mathrm{B} 97 \mathrm{X}-\mathrm{D}$ functional is range-separated and includes dispersion correction, the energy of the QM part was recalculated using single-point CBS/DLPNO-CCSD(T) calculations. This level of theory should remove possible BSSE effects. Inclusion of ZPE effect is also important, even for atoms.

This protocol was applied to both crystalline and amorphous ices. For the latter, eight snapshots obtained from classical MD calculations were selected. This allows us to propose accurate binding energies for the set of studied species. The dispersion of the BE's is rather large, reflecting a large diversity in the hydrogen bond network and the possible inclusion of the adsorbate on the surface. In the case of carbon atom, two different pathways were identified, depending on the local environment. One of them leads to the formation of a covalently bonded $\mathrm{COH}_{2}$ molecule while the other one, less frequent, produces a $\mathrm{COH}^{-} \mathrm{H}_{3} \mathrm{O}^{+}$complex. 


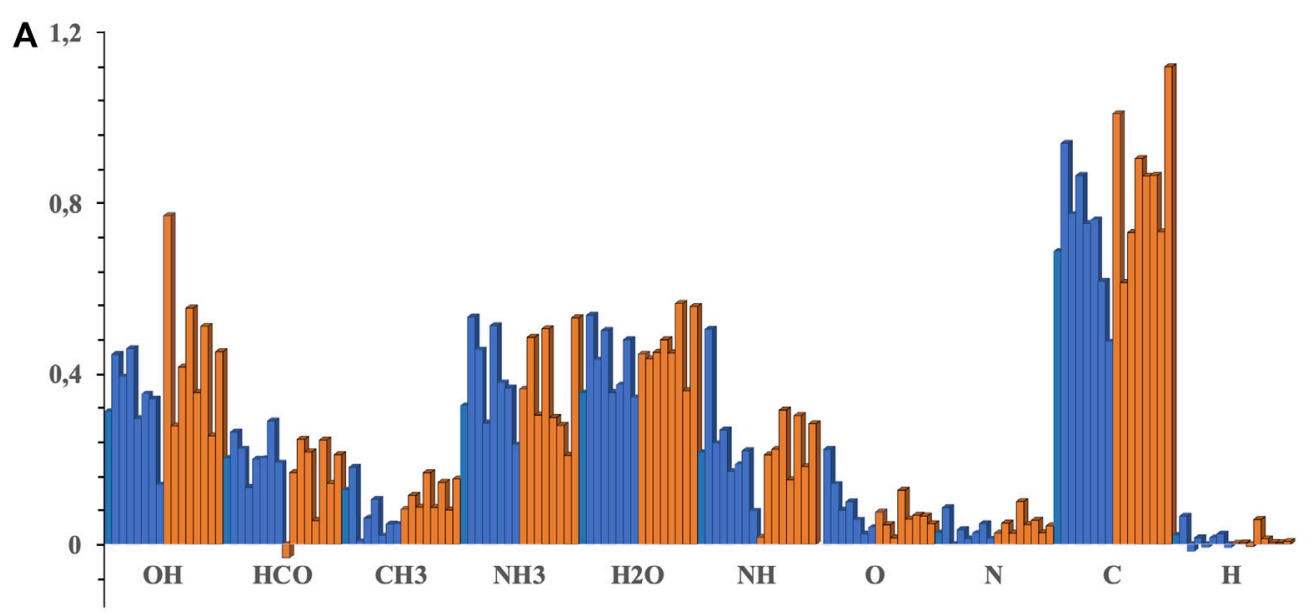

B

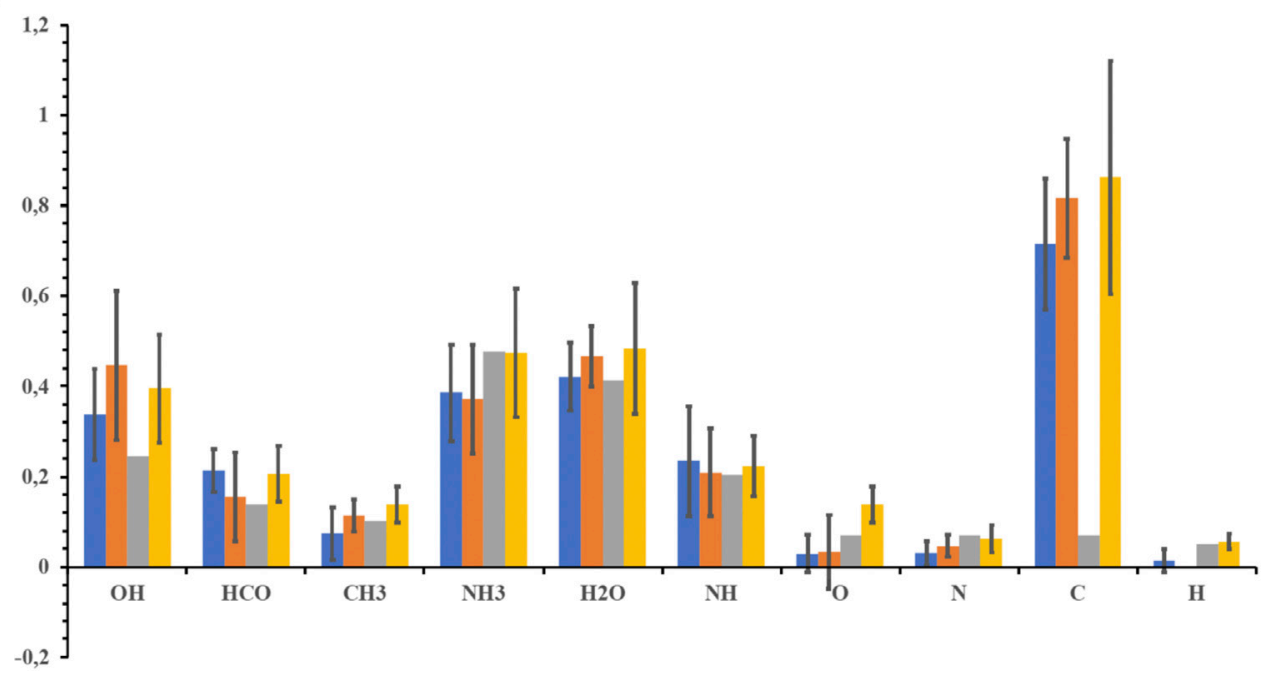

FIGURE 11 | (A) Summary of CBS BE (in eV) distributions for crystalline (blue) and amorphous (orange) ices (B) Comparison of calculated average BE's (with standard deviation) between crystalline (blue) and amorphous (orange) ice. Values, when available, taken from UMIST (McElroy et al., 2013) and KIDA (Wakelam et al., 2012) databases are also represented in grey and yellow, respectively.

The present method is sufficiently versatile to be applied to other systems, including larger adsorbed molecules and/or, mixed ices. The accuracy can also be further benchmarked by adapting the choice of the functional and basis set. A possible extension would be to use a three-layer ONIOM. The computational cost of the DLPNO-CCSD(T) single point calculations can also be limited by using only the def2-TZVPP basis set, for example. It would also be interesting to increase the number of studied snapshots in order to get more statistically significant values. Finally, reactive processes may also be tractable.

As a conclusion, the present study brings new BEs estimations to be included in astrochemical models. When pure quantum chemistry calculations are performed, a single and unique value is produced that corresponds to the global minimum. With the methodology proposed here, the statistics over different configurations provides a set of values: maximum, minimum, average and standard deviation, $\mathrm{BE}$ distribution. The average $\mathrm{BE}$ accounts for the diversity of adsorption sites, whereas using the maximum BE implies that adsorption occurs preferentially in the most stable sites. An extensive investigation should be carried out to quantify how sensitive the astrochemical models are with respect to $\mathrm{BE}$ variations. This is beyond the scope of the present study but would help to target the BE accuracy and consequently to adjust the theoretical methodology accordingly.

\section{DATA AVAILABILITY STATEMENT}

The original contributions presented in the study are included in the article/Supplementary Material, further inquiries can be directed to the corresponding authors. 


\section{AUTHOR CONTRIBUTIONS}

DD did most of the quantum chemical calculations and wrote parts of the manuscripts. CT did the classical dynamics calculations and participated in the writing. MM helped writing the manuscript.

\section{FUNDING}

The authors acknowledge support from the CaPPA project (Chemical and Physical Properties of the Atmosphere) funded by the French National Research Agency (ANR) through the PIA (Programme d'Investissement d'Avenir) under Contract No. ANR-10-LABX-005; the Région Hauts de France and the Ministère de l'Enseignement Supérieur et de la Recherche (CPER Climibio) and the European Fund for Regional Economic Development for their financial support. This work

\section{REFERENCES}

Abascal, J. L., and Vega, C. (2005). A general purpose model for the condensed phases of water: TIP4P/2005. J. Chem. Phys. 123 (23), 234505. doi:10.1063/1. 2121687

Al-Halabi, A., and Van Dishoeck, E. F. (2007). Hydrogen adsorption and diffusion on amorphous solid water ice. Mon. Notices Royal Astron. Soc. 382 (4), 1648-1656. doi:10.1111/j.1365-2966.2007.12415.x

Bolina, A. S., Wolff, A. J., and Brown, W. A. (2005). Reflection absorption infrared spectroscopy and temperature-programmed desorption studies of the adsorption and desorption of amorphous and crystalline water on a graphite surface. J. Phys. Chem. B. 109 (35), 16836-16845. doi:10.1021/ jp0528111

Buch, V., and Devlin, J. P. (1991). Spectra of dangling OH bonds in amorphous ice: assignment to 2 and 3-coordinated surface molecules. J. Chem. Phys. 94 (5), 4091. doi:10.1063/1.460638

Burke, D. J., and Brown, W. A. (2010). Ice in space: surface science investigations of the thermal desorption of model interstellar ices on dust grain analogue surfaces. Phys. Chem. Chem. Phys. 12 (23), 5947. doi:10.1039/b917005g

Chai, J. D., and Head-Gordon, M. (2008). Long-range corrected hybrid density functionals with damped atom-atom dispersion corrections. Phys. Chem. Chem. Phys. 10 (44), 6615-6620. doi:10.1039/b810189b

Chung, L. W., Sameera, W. M., Ramozzi, R., Page, A. J., Hatanaka, M., Petrova, G. P., et al. (2015). The ONIOM method and its applications. Chem. Rev. 115 (12), 5678-5796. doi:10.1021/cr5004419

Clark, T., Chandrasekhar, J., Spitznagel, G. N. W., and Schleyer, P. V. R. (1983). Efficient diffuse function-augmented basis sets for anion calculations. III. The 3-21 +G basis set for first-row elements, Li-F. J. Comput. Chem. 4 (3), 294-301. doi:10.1002/jcc.540040303

Collings, M. P., Anderson, M. A., Chen, R., Dever, J. W., Viti, S., Williams, D. A., et al. (2004). A laboratory survey of the thermal desorption of astrophysically relevant molecules. Mon. Notices Royal Astron. Soc. 354 (4), 1133-1140. doi:10. 1111/j.1365-2966.2004.08272.x

Cuppen, H. M., Walsh, C., Lamberts, T., Semenov, D., Garrod, R. T., Penteado, E. M., et al. (2017). Grain surface models and data for astrochemistry. Space Sci. Rev. 212 (1-2), 1-58. doi:10.1007/s11214-016-0319-3

Das, A., Sil, M., Gorai, P., Chakrabarti, S. K., and Loison, J. C. (2018). An approach to estimate the binding energy of interstellar species. Astrophys. J. Suppl. Ser. 237 (1), 9. doi:10.3847/1538-4365/aac886

David, V., Grinberg, N., Moldoveanu, S. C., Grinberg, N., and Moldoveanu, S. C. (2017). Long-range molecular interactions involved in the retention mechanisms of liquid chromatography. Adv. Chromatogr. 54, 73-110. doi: 10.1201/9781315116372-4. was performed using HPC resources from GENCI-TGCC (Grant No. 2020-A0050801859).

\section{ACKNOWLEDGMENTS}

The authors thank the PCMI (Programme National de Physique et Chimie du Milieu Interstellaire) for support and the Centre de Ressources Informatiques (CRI) of the Universite of Lille for providing computing time. Special thanks to A. Rivero-Santamaria for useful discussions and J. C. Loison and V. Wakelam for their insightful comments.

\section{SUPPLEMENTARY MATERIAL}

The Supplementary Material for this article can be found online at: https://www.frontiersin.org/articles/10.3389/fspas.2021.645243/ full\#supplementary-material.

Ditchfield, R., Hehre, W. J., and Pople, J. A. (1971). Self-consistent molecularorbital methods. IX. An extended Gaussian-type basis for molecular-orbital studies of organic molecules. J. Chem. Phys. 54, 724-728. doi:10.1063/1.1674902

Dohnálek, Z., Kimmel, G. A., Ayotte, P., Smith, R. S., and Kay, B. D. (2003). The deposition angle-dependent density of amorphous solid water films. J. Chem. Phys. 118 (1), 364. doi:10.1063/1.1525805

Du, S., Francisco, J. S., Schenter, G. K., Iordanov, T. D., Garrett, B. C., Dupuis, M., et al. (2006). The $\mathrm{OH}$ radical $-\mathrm{H}_{2} \mathrm{O}$ molecular interaction potential. J. Chem. Phys. 124, 224318-224415. doi:10.1063/1.2200701

Dulieu, F., Congiu, E., Noble, J., Baouche, S., Chaabouni, H., Moudens, A., et al. (2013). How micron-sized dust particles determine the chemistry of our universe. Sci. Rep. 3 (1), 1338. doi:10.1038/srep01338

Duvernay, F., Rimola, A., Theule, P., Danger, G., Sanchez, T., and Chiavassa, T. (2014). Formaldehyde chemistry in cometary ices: the case of $\mathrm{HOCH}_{2} \mathrm{OH}$ formation. Phys. Chem. Chem. Phys. 16 (44), 24200-24208. doi:10.1039/ c4cp03031a

Ehrenfreund, P., and Charnley, S. B. (2000). Organic molecules in the interstellar medium, comets, and meteorites: a voyage from dark clouds to the early Earth. Annu. Rev. Astron. Astrophys. 38 (1), 427-483. doi:10.1146/annurev.astro.38. 1.427

Ehrenfreund, P., Fraser, H. J., Blum, J., Cartwright, J. H. E., Garcia Ruiz, J. M., Hadamcik, E., et al. (2003). Physics and chemistry of icy particles in the universe: answers from microgravity. Planet. Space Sci. 51 (7-8), 473-494. doi:10.1016/s0032-0633(03)00052-7

Enrique-Romero, J., Rimola, A., Ceccarelli, C., Ugliengo, P., Balucani, N., and Skouteris, D. (2019). Reactivity of $\mathrm{HCO}$ with $\mathrm{CH}_{3}$ and $\mathrm{NH}_{2}$ on water ice surfaces. a comprehensive accurate quantum chemistry study. ACS Earth Space Chem. 3 (10), 2158-2170. doi:10.1021/acsearthspacechem.9b00156

Ferrero, S., Martínez-Bachs, B., Enrique-Romero, J., Rimola, A., et al. (2020a). "Adsorption of atoms on a crystalline ice surface model: results from periodic ab initio simulations," in Computational science and its applications-ICCSA 2020. Editors O. Gervasi, B. Murgante, S. Misra, C. Garau, I. Blečić, and D. Taniar (Cham, Switerzland: Springer international publishing), 553-560.

Ferrero, S., Zamirri, L., Ceccarelli, C., Witzel, A., Rimola, A., and Ugliengo, P. (2020b). Binding energies of interstellar molecules on crystalline and amorphous models of water ice by ab-initio calculations. Astrophys. J. 904 (1), 904-911. doi:10.3847/1538-4357/abb953

Fraser, H. J., Collings, M. P., McCoustra, M. R. S., and Williams, D. A. (2001). Thermal desorption of water ice in the interstellar medium. Mon. Notices Royal Astron. Soc. 327 (4), 1165-1172. doi:10.1046/j.1365-8711.2001.04835.x

Frisch, M. J., Trucks, G. W., Schlegel, H. B., Scuseria, G. E., Robb, M. A., Cheeseman, J. R., et al. (2016). Gaussian 16 Rev. B.01. Wallingford, CT: Gaussian Inc. 
Garrod, R., Park, I. H., Caselli, P., and Herbst, E. (2006). Are gas-phase models of interstellar chemistry tenable? the case of methanol. Faraday Discuss. 133, 51. doi:10.1039/b516202e

Garrod, R. T., and Herbst, E. (2006). Formation of methyl formate and other organic species in the warm-up phase of hot molecular cores. Astron. Astrophys. 457 (3), 927-936. doi:10.1051/0004-6361:20065560

Goumans, T. P., and Kästner, J. (2010). Hydrogen-atom tunneling could contribute to $\mathrm{H}_{2}$ formation in space. Angew. Chem. Int. Ed. Engl. 49 (40), 7350-7352. doi:10.1002/anie.201001311

Guedes, R. C., Costa Cabral, B. J., Martinho Simões, J. A., and Cabral do Couto, P. (2003). The hydration of the $\mathrm{OH}$ radical: microsolvation modeling and statistical mechanics simulation. J. Chem. Phys. 119 (14), 7344-7355. doi:10. 1063/1.1605939.

Guo, Y., Riplinger, C., Becker, U., Liakos, D. G., Minenkov, Y., Cavallo, L., et al. (2018). Communication: an improved linear scaling perturbative triples correction for the domain based local pair-natural orbital based singles and doubles coupled cluster method [DLPNO-CCSD(T)]. J. Chem. Phys. 148 (1), 011101. doi:10.1063/1.5011798

Hama, T., and Watanabe, N. (2013). Surface processes on interstellar amorphous solid water: adsorption, diffusion, tunneling reactions, and nuclear-spin conversion. Chem. Rev. 113, 8783-8839. doi:10.1021/cr4000978

Hariharan, P. C., and Pople, J. A. (1973). The influence of polarization functions on molecular orbital hydrogenation energies. Theoret. Chim. Acta 28, 213-222. doi:10.1007/bf00533485

Haynes, D. R., Tro, N. J., and George, S. M. (1992). Condensation and evaporation of water on ice surfaces. J. Phys. Chem. 96 (21), 8502-8509. doi:10.1021/ j100200a055

He, J., Jing, D., and Vidali, G. (2014). Atomic oxygen diffusion on and desorption from amorphous silicate surfaces. Phys. Chem. Chem. Phys. 16 (8), 3493-3500. doi:10.1039/c3cp54328e

He, J., Shi, J., Hopkins, T., Vidali, G., and Kaufman, M. J. (2015). A new determination of the binding energy of atomic oxygen on dust grain surfaces: experimental results and simulations. Astrophys. J. 801 (2), 120. doi:10.1088/0004-637x/801/2/120

Hehre, W. J., Ditchfield, R., and Pople, J. A. (1972). Self-consistent molecular orbital methods. XII. Further extensions of Gaussian-type basis sets for use in molecular orbital studies of organic molecules. J. Chem. Phys. 56, 2257-2261. doi:10.1063/1.1677527

Hellweg, A., Hättig, C., Höfener, S., and Klopper, W. (2007). Optimized accurate auxiliary basis sets for RI-MP2 and RI-CC2 calculations for the atoms Rb to Rn. Theor. Chem. Acc. 117 (4), 587-597. doi:10.1007/s00214-007-0250-5

Hickson, K. M., Loison, J. C., Nuñez-Reyes, D., and Méreau, R. (2016). Quantum tunneling enhancement of the $\mathrm{C}+\mathrm{H}_{2} \mathrm{O}$ and $\mathrm{C}+\mathrm{D}_{2} \mathrm{O}$ reactions at low temperature. J. Phys. Chem. Lett. 7 (18), 3641-3646. doi:10.1021/acs.jpclett. 6b01637

Ioppolo, S., van Boheemen, Y., Cuppen, H. M., van Dishoeck, E. F., and Linnartz, H. (2011). Surface formation of $\mathrm{CO}_{2}$ ice at low temperatures. Mon. Notices Royal Astron. Soc. 413 (3), 2281-2287. doi:10.1111/j.1365-2966.2011.18306.x

Karssemeijer, L. J., Pedersen, A., Jónsson, H., and Cuppen, H. M. (2012). Longtimescale simulations of diffusion in molecular solids. Phys. Chem. Chem. Phys. 14 (31), 10844-10852. doi:10.1039/c2cp41634d

Kimmel, G. A., Dohnálek, Z., Stevenson, K. P., Smith, R. S., and Kay, B. D. (2001). Control of amorphous solid water morphology using molecular beams. II. Ballistic deposition simulations. J. Chem. Phys. 114 (12), 5295-5303. doi:10. 1063/1.1350581

Koch, D. M., Toubin, C., Peslherbe, G. H., and Hynes, J. T. (2008). A theoretical study of the formation of the aminoacetonitrile precursor of glycine on icy grain mantles in the interstellar medium. J. Phys. Chem. C 112 (8), 2972-2980. doi:10. 1021/jp076221+

Koch, D. M., Toubin, C., Xu, S., Peslherbe, G. H., and Hynes, J. T. (2007). Concerted proton-transfer mechanism and solvation effects in the $\mathrm{HNC} / \mathrm{HCN}$ isomerization on the surface of icy grain mantles in the interstellar medium. J. Phys. Chem. C 111 (41), 15026-15033. doi:10. $1021 /$ jp076220h

Liakos, D. G., Sparta, M., Kesharwani, M. K., Martin, J. M., and Neese, F. (2015). Exploring the accuracy limits of local pair natural orbital coupledcluster theory. J. Chem. Theory Comput. 11 (4), 1525-1539. doi:10.1021/ ct501129s
McElroy, D., Walsh, C., Markwick, A. J., Cordiner, M. A., Smith, K., and Millar, T. J. (2013). The UMIST database for astrochemistry 2012. Astron. Astrophys. 550, 13. doi:10.1051/0004-6361/201220465

Michoulier, E., Noble, J. A., Simon, A., Mascetti, J., and Toubin, C. (2018). Adsorption of PAHs on interstellar ice viewed by classical molecular dynamics. Phys. Chem. Chem. Phys. 20 (13), 8753-8764. doi:10.1039/ c8cp00593a

Michoulier, E., Toubin, C., Simon, A., Mascetti, J., Aupetit, C., and Noble, J. A. (2020). Perturbation of the surface of amorphous solid water by the adsorption of polycyclic aromatic hydrocarbons. J. Phys. Chem. C 124 (5), 2994-3001. doi:10.1021/acs.jpcc.9b09499

Minissale, M., Congiu, E., and Dulieu, F. (2016). Direct measurement of desorption and diffusion energies of $\mathrm{O}$ and $\mathrm{N}$ atoms physisorbed on amorphous surfaces. Astron. Astrophys. 585, A146. doi:10.1051/0004-6361/201526702

Minissale, M., Loison, J-C., Baouche, S., Chaabouni, H., Congiu, E., and Dulieu, F. (2015). Solid-state formation of $\mathrm{CO}_{2}$ via the $\mathrm{H}_{2} \mathrm{CO}+\mathrm{O}$ reaction. Astron. Astrophys. 577, A2. doi:10.1051/0004-6361/201424342

Miyazaki, A., Watanabe, N., Sameera, W. M. C., Nakai, Y., Tsuge, M., Hama, T., et al. (2020). Photostimulated desorption of $\mathrm{OH}$ radicals from amorphous solid water: evidence for the interaction of visible light with an $\mathrm{OH}$-ice complex. Phys. Rev. A 102 (5). 052822. doi:10.1103/PhysRevA.102.052822.

Molpeceres, G., Zaverkin, V., and Kästner, J. (2020). Neural-network assisted study of nitrogen atom dynamics on amorphous solid water-I. adsorption and desorption. Mon. Notices Royal Astron. Soc. 499 (1), 1373-1384. doi:10. 1093/mnras/staa2891

Neese, F., and Valeev, E. F. (2011). Revisiting the atomic natural orbital approach for basis sets: robust systematic basis sets for explicitly correlated and conventional correlated $a b$ initio methods? J. Chem. Theory Comput. 7 (1), 33-43. doi:10.1021/ct100396y

Neese, F., Wennmohs, F., Becker, U., and Riplinger, C. (2020). The ORCA quantum chemistry program package. J. Chem. Phys. 152 (22), 224108. doi:10.1063/5.0004608

Neese, F. (2018). Software update: the ORCA program system, version 4.0. WIREs Comput. Mol. Sci. 8 (1), e1327. doi:10.1002/wcms.1327

Noble, J. A., Congiu, E., Dulieu, F., and Fraser, H. J. (2012). Thermal desorption characteristics of $\mathrm{CO}, \mathrm{O}_{2}$, and $\mathrm{CO}_{2}$ on non-porous water, crystalline water and silicate surfaces at sub-monolayer and multilayer coverages. Mon. Notices Royal Astron. Soc. 421, 768-779. doi:10.1111/j.1365-2966.2011.20351.x

Noble, J. A., Martin, C., Fraser, H. J., Roubin, P., and Coussan, S. (2014). Unveiling the surface structure of amorphous solid water via selective infrared irradiation of OH stretching modes. J. Phys. Chem. Lett. 5 (5), 826-829. doi:10.1021/ jz5000066

Penteado, E. M., Walsh, C., and Cuppen, H. M. (2017). Sensitivity analysis of grain surface chemistry to binding energies of ice species. Astrophys. J. 844 (1), 71. doi:10.3847/1538-4357/aa78f9

Peters, P. S., Duflot, D., Wiesenfeld, L., and Toubin, C. (2013). The H + CO $\rightleftharpoons$ HCO reaction studied by $a b$ initio benchmark calculations. J. Chem. Phys. 139 (16), 164310. doi:10.1063/1.4826171

Pirim, C., and Krim, L. (2011). An FTIR study on the catalytic effect of water molecules on the reaction of $\mathrm{CO}$ successive hydrogenation at $3 \mathrm{~K}$. Chem. Phys. 380 (1-3), 67-76. doi:10.1016/j.chemphys.2010.12.008

Pontoppidan, K. M., Boogert, A. C. A., Fraser, H. J., van Dishoeck, E. F., Blake, G. A., Lahuis, F., et al. (2008). The c2d Spitzer Spectroscopic survey of ices around low-mass young stellar objects. II. $\mathrm{CO}_{2}$. Astrophys. J. 678 (2), 1005. doi:10.1086/ 533431

Rimola, A., Taquet, V., Ugliengo, P., Balucani, N., and Ceccarelli, C. (2014). Combined quantum chemical and modeling study of $\mathrm{CO}$ hydrogenation on water ice. Astron. Astrophys. 572, A70. doi:10.1051/0004-6361/201424046

Rimola, A., and Ugliengo, P. (2009). The role of defective silica surfaces in exogenous delivery of prebiotic compounds: clues from first principles calculations. Phys. Chem. Chem. Phys. 11 (14), 2497-2506. doi:10.1039/ b820577a

Ruaud, M., Wakelam, V., and Hersant, F. (2016). Gas and grain chemical composition in cold cores as predicted by the Nautilus three-phase model. Mon. Notice Royal Astron. Soc. 459 (4), 3756-3767. doi:10.1093/mnras/stw887

Sameera, W. M. C., and Maseras, F. (2018). Expanding the range of force fields available for ONIOM calculations: the SICTWO interface. J. Chem. Inf. Model. 58 (9), 1828-1835. doi:10.1021/acs.jcim.8b00332 
Sameera, W. M. C., Senevirathne, B., Andersson, S., Al-lbadi, M., Hidaka, H., Kouchi, A., et al. (2021). $\mathrm{CH}_{3} \mathrm{O}$ radical binding on hexagonal water ice and amorphous solid water. J. Phys. Chem. A 125 (1), 387-393. doi:10.1021/acs.jpca. 0c09111

Sameera, W. M. C., Senevirathne, B., Andersson, S., Maseras, F., and Nyman, G. (2017). ONIOM(QM:AMOEBA09) study on binding energies and binding preference of $\mathrm{OH}, \mathrm{HCO}$, and $\mathrm{CH}_{3}$ radicals on hexagonal water ice (Ih). J. Phys. Chem. C 121 (28), 15223-15232. doi:10.1021/acs.jpcc.7b04105

Sandford, S. A., and Allamandola, L. J. (1988). The condensation and vaporization behavior of $\mathrm{H}_{2} \mathrm{O}$ : $\mathrm{CO}$ ices and implications for interstellar grains and cometary activity. Icarus 76 (2), 201-224. doi:10.1016/0019-1035(88)90069-3

Schlegel, H. B., and McDouall, J. J. W. (1991). "Do you have SCF stability and convergence problems? Molecular structure and reactivity," in Computational advances in organic chemistry. Editors C. Ögretir and I. G. Csizmadia (Dordrecht: NATO ASI Series), 167-185.

Shimonishi, T., Nakatani, N., Furuya, K., and Hama, T. (2018). Adsorption energies of carbon, nitrogen, and oxygen atoms on the low-temperature amorphous water ice: a systematic estimation from quantum chemistry calculations. Astrophys. J. 855 (1), 27. doi:10.3847/1538-4357/aaaa6a

Stewart, J. J. P. (2007). Optimization of parameters for semiempirical methods V: modification of NDDO approximations and application to 70 elements. J. Mol. Model. 13 (12), 1173-1213. doi:10.1007/s00894-007-0233-4

Taquet, V., Peters, P. S., Kahane, C., Ceccarelli, C., López-Sepulcre, A., Toubin, C., et al. (2013). Water ice deuteration: a tracer of the chemical history of protostars. Astron. Astrophys. 550, A127. doi:10.1051/0004-6361/201220084

Throssell, K., and Frisch, M. J. (Forthcoming 2018). Evaluation and Improvement of Semi-empirical methods I: PM7R8: a variant of PM7 with numerically stable hydrogen bonding corrections. doi:10.14418/wes01.3.76

Throssell, K. (2017). Evaluating and approximate, improving LCAO-MO theory with restored overlap and bond order bond energy corrections. MS dissertation. Middletown (Connecticut): Wesleyan University.

Ulbricht, H., Zacharia, R., Cindir, N., and Hertel, T. (2006). Thermal desorption of gases and solvents from graphite and carbon nanotube surfaces. Carbon 44 (14), 2931-2942. doi:10.1016/j.carbon.2006.05.040

van Dishoeck, E. F. (1998). What can ISO tell us about gas-grain chemistry?. Faraday Disc. 109, 31-46. doi:10.1039/a800815i

Vega, C., and Abascal, J. L. (2011). Simulating water with rigid non-polarizable models: a general perspective. Phys. Chem. Chem. Phys. 13 (44), 19663-19688. doi:10.1039/c1cp22168j

Wakelam, V., Herbst, E., Loison, J.-C., Smith, I. W. M., Chandrasekaran, V., Pavone, B., et al. (2012). A KInetic database for astrochemistry (KIDA). Astrophys. J., Suppl. Ser. 199 (1), 21. doi:10.1088/0067-0049/199/1/21
Wakelam, V., Loison, J.-C., Mereau, R., and Ruaud, M. (2017). Binding energies: new values and impact on the efficiency of chemical desorption. Mol. Astrophys. 6, 22-35. doi:10.1016/j.molap.2017.01.002

Weigend, F., and Ahlrichs, R. (2005). Balanced basis sets of split valence, triple zeta valence and quadruple zeta valence quality for $\mathrm{H}$ to $\mathrm{Rn}$ : design and assessment of accuracy. Phys. Chem. Chem. Phys. 7 (18), 3297-3305. doi:10.1039/b508541a

Weigend, F. (2008). Hartree-Fock exchange fitting basis sets for $\mathrm{H}$ to $\mathrm{Rn}$. J. Comput. Chem. 29 (2), 167-175. doi:10.1002/jcc.20702

Weigend, F., Kattannek, M., and Ahlrichs, R. (2009). Approximated electron repulsion integrals: cholesky decomposition versus resolution of the identity methods. J. Chem. Phys. 130 (16), 164106. doi:10.1063/1.3116103

Weinhold, F., and Carpenter, J. E. (1988). “The natural bond orbital lewis structure concept for molecules, radicals, and radical ions," in The structure of small molecules and ions. Editors R. Naaman and Z. Vager (Boston, MA: Springer United States), 227-236.

Whittet, D. C. B., Schutte, W. A., Tielens, A., Boogert, A. C. A., de Graauw, T., Ehrenfreund, P., et al. (1996). An ISO SWS view of interstellar ices: first results. Astron. Astrophys. 315, L357-L360.

Wiberg, K. B. (1968). Application of the pople-santry-segal CNDO method to the cyclopropylcarbinyl and cyclobutyl cation and to bicyclobutane. Tetrahedron 24 (3), 1083-1096. doi:10.1016/0040-4020(68)88057-3

Yocum, K. M., Smith, H. H., Todd, E. W., Mora, L., Gerakines, P. A., Milam, S. N., et al. (2019). Millimeter/submillimeter spectroscopic detection of desorbed ices: a new technique in laboratory astrochemistry. J. Phys. Chem. A. 123 (40), 8702-8708. doi:10.1021/acs.jpca.9b04587

Zhao, Y., and Truhlar, D. G. (2008). The M06 suite of density functionals for main group thermochemistry, thermochemical kinetics, noncovalent interactions, excited states, and transition elements: two new functionals and systematic testing of four M06-class functionals and 12 other functionals. Theor. Chem. Account. 120 (1), 215-241. doi:10.1007/s00214-007-0310-x

Conflict of Interest: The authors declare that the research was conducted in the absence of any commercial or financial relationships that could be construed as a potential conflict of interest.

Copyright (C) 2021 Duflot, Toubin and Monnerville. This is an open-access article distributed under the terms of the Creative Commons Attribution License (CC BY). The use, distribution or reproduction in other forums is permitted, provided the original author(s) and the copyright owner(s) are credited and that the original publication in this journal is cited, in accordance with accepted academic practice. No use, distribution or reproduction is permitted which does not comply with these terms. 